\title{
HÖLDER CONTINUITY OF THE MINIMIZER OF AN OBSTACLE PROBLEM WITH GENERALIZED ORLICZ GROWTH
}

\author{
ARTTU KARPPINEN, MIKYOUNG LEE
}

\begin{abstract}
We prove local $C^{0, \alpha}$ - and $C^{1, \alpha}$-regularity for the local solution to an obstacle problem with non-standard growth. These results cover as special cases standard, variable exponent, double phase and Orlicz growth.
\end{abstract}

\section{INTRODUCTION}

The classical obstacle problem is motivated by the description of the equilibrium position of an elastic membrane lying above an obstacle. Its mathematical interpretation is to find minimizers of the elastic energy functional with the addition of a constraint that presents the obstacle. This model leads to the mathematical objects called variational inequalities. In this regard, obstacle problem is deeply related to the study of the calculus of variation and the partial differential equation. It arises in broad applications, such as the study of fluid filtration in porous media, constrained heating, elasto-plasticity, optimal control, and financial mathematics.

The fundamental problem that appears with the study of the obstacle problem is to find the optimal regularity of minimizers. The primary model we have in mind is the nonautonomous minimization problem

$$
\min _{u}\left\{\int_{\Omega} F(x, \nabla u) d x: u \geqslant \psi \text { a.e. in } \Omega,\left.u\right|_{\partial \Omega}=g\right\}
$$

where $\Omega$ is a bounded open set in $\mathbb{R}^{n}$ and $\psi$ is the obstacle. If $u$ satisfies (1.1), we say that $u$ is a solution to the obstacle problem. In this paper, we are interested in Hölder regularity properties of solutions to the obstacle problems related to (1.1) with nonstandard growth conditions. In fact, the regularity results for such obstacle problems have been achieved by applying results and techniques developed in the research on the unconstrained case, i.e. when $\psi=-\infty$.

For the unconstrained case, there have been many extensive researches on the regularity theory, including $C^{0, \alpha}$ - and $C^{1, \alpha}$-regularity, for the nonstandard growth problem such as the non-autonomous minimization problem (1.1) when $F$ satisfies $(p, q)$-growth conditions, that is, $|z|^{p} \lesssim F(x, z) \lesssim|z|^{q}+1, p<q$, starting with Marcellini's seminal papers [40, 41]. Several model functionals mainly in relation to the Lavrentiev phenomenon were proposed by Zhikov [51] in order to describe the behavior of anisotropic materials in the framework of homogenization and nonlinear elasticity. The main feature of such functionals, including

Date: February 25, 2021.

2010 Mathematics Subject Classification. 35B65; 49N60, 35A15, 46E35.

Key words and phrases. Hölder continuity, minimizer, obstacle problem, generalized Orlicz space, Musielak-Orlicz spaces, nonstandard growth.

A. Karppinen was partially supported by Turku University Foundation. M. Lee was supported by the National Research Foundation of Korea (NRF) grant funded by the Korea Government (NRF2019R1F1A1061295). 
the variable exponent functionals with

$$
F(x, z) \approx|z|^{p(x)} \text { for } 1<\inf _{\Omega} p(x) \leqslant \sup _{\Omega} p(x)<\infty,
$$

and the double phase functionals with

$$
F(x, z) \approx|z|^{p}+a(x)|z|^{q} \text { for } 1<p \leqslant q<\infty \text { and } a(\cdot) \geqslant 0
$$

is that those integrands $F(x, z)$ change their ellipticity and growth properties according to the point $x$. Starting with a higher integrability result of Zhikov [52], regularity problems for minimizers of the variable exponent functionals have been vigorously studied in for instance $[1,14,19,23]$ (see also references in the survey [44]). For the double phase functionals, the regularity theory was developed by Baroni, Colombo and Mingione in a series of remarkable papers $[3,4,13]$. We also refer to $[15,17,28,45,49,50]$ for regularity results in the various variants and borderline cases. Maximal regularity for Orlicz growth was settled by Lieberman in [39] and for Hölder continuity of the solution assumptions have been relaxed in [2]. Other regularity results for Orlicz growth can be found for example in $[11,12,18]$. Furthermore, regularity properties have been recently studied for minimizers of the functionals with the generalized Orlicz growth that cover the functionals referred above, see for instance $[32,33,34]$.

Our first main result, Theorem 2.6, concerns local $C^{0, \alpha}$-continuity of the solution for some $\alpha \in(0,1)$. This standard Hölder continuity of solution to an obstacle problem is relevant by the side of maximal regularity results since less assumptions are needed for the functional and the obstacle. We do not require differentiability of the functional and the obstacle is assumed to be merely Hölder continuous rather than having continuous gradient. These lighter results are applied for example in study of removable sets [8, 38].

In the very recent paper [36], $C^{0, \alpha}$ - and $C^{1, \alpha}$ - regularity properties were established by Hästö-Ok for minimizers of non-autonomous functionals with sharp and general conditions, covering all the previous results. It is worth pointing out that this result assumed no gap between growth exponents $p$ and $q$. Instead their work is based on a carefully crafted continuity assumption (wVA1) for the $x$ variable in $\varphi$. This assumption turns out to capture sharp structural assumptions, which ensure regularity, in the important special cases such as double phase and variable exponent cases. Following their results and ideas, we are concerned with the regularity properties for solutions of the obstacle problems under $(p, q)$-growth condition. These results are collected in Theorem 2.7.

For the obstacle problem, it is noted that the solution inherits the regularity properties from the obstacle. In particular, for the linear obstacle problem, i.e., when $F(x, z) \approx|z|^{2}$ in (1.1), it is well known that the solution has the same regularity as the obstacle $\psi$, see for instance $[5,7]$. However this is not usually permitted in the nonlinear cases. Hence extensive research in this direction has been done into the regularity of solutions to the nonlinear obstacle problems. As the first result for the nonlinear functional with standard growth, i.e. when $F(x, z) \approx|z|^{p}$ with $1<p<\infty$, Michael-Ziemer [43] proved that the solution is Hölder continuous when the obstacle $\psi$ is Hölder continuous. Choe [9] established the same result for the gradient of solutions when the gradient of the obstacle $\psi$ is Hölder continuous. We further refer to $[10,24,25,46,48]$ for the Hölder regularity results on the nonlinear obstacle problems related to $p$-Laplace type functionals and more general functionals. Concerning with the nonstandard growth cases, Hölder type regularity results for obstacle problems with $p(x)$-growth were obtained in several papers for instance [20, 21, 22]. The $L^{p(\cdot)} \log L$-growth case, which can be regarded as a borderline case lying between Orlicz growth and variable exponent growth, was considered in the recent paper [47]. Hölder regularity results for the double phase case have been studied for example in [8]. 
Obstacle problems for functionals having $(p, q)$ growth have been studied in for example $[6,16]$ with different assumptions. We do not assume any gap between exponents $p$ and $q$ or $C^{2}$-regularity of our functional, but instead work with the (wVA1) condition. Results of this paper cover all the previous results in the nonstandard growth cases mentioned above. We would like to point out that our results are new in many borderline cases including the double phase case.

\section{PRELIMINARIES AND MAIN RESUlTS}

By $\Omega \subset \mathbb{R}^{n}$ we denote a bounded domain, i.e. an open and connected. If for an open set $\Omega^{\prime}$ we have $\overline{\Omega^{\prime}} \subset \Omega$, we denote it simply as $\Omega^{\prime} \Subset \Omega$. By $Q_{r}$ we mean a cube with side length $2 r$ and by $B_{r}$ a ball with radius $r$. For an integrable function $g: U \subset \mathbb{R}^{n} \rightarrow \mathbb{R}$, we define the average of $g$ in $U$ by

$$
\bar{g}_{U}:=f_{U} g d x=\frac{1}{|U|} \int_{U} g d x
$$

Generalized $\Phi$-functions. In this section we introduce the basic notations, definitions and assumptions for our growth rate.

A function $f$ is said to be almost increasing if there exists $L \geqslant 1$ such that $f(s) \leqslant L f(t)$ for all $s \leqslant t$. Almost decreasing is defined analogously. By increasing we mean that the inequality holds for $L=1$ (some call this non-decreasing), similarly for decreasing.

Definition 2.1. We say that $\varphi: \Omega \times[0, \infty) \rightarrow[0, \infty]$ is a (generalized) $\Phi$-prefunction if the following hold:

(i) The function $x \mapsto \varphi(x, t)$ is measurable for every $t \in[0, \infty)$.

(ii) The function $t \mapsto \varphi(x, t)$ is non-decreasing for every $x \in \Omega$.

(iii) $\lim _{t \rightarrow 0^{+}} \varphi(x, t)=\varphi(x, 0)=0$ and $\lim _{t \rightarrow \infty} \varphi(x, t)=\infty$ for every $x \in \Omega$.

A $\Phi$-prefunction $\varphi$ is called a (generalized weak) $\Phi$-function, denoted by $\varphi \in \Phi_{w}(\Omega)$, if the function $t \mapsto \frac{\varphi(x, t)}{t}$ is almost increasing in $(0, \infty)$ for every $x \in \Omega$, and a (generalized) convex $\Phi$-function, denoted by $\varphi \in \Phi_{c}(\Omega)$, if the function $t \mapsto \varphi(x, t)$ is left-continuous and convex for every $x \in \Omega$. Additionally, we denote $\varphi \in \Phi_{w}$ or $\varphi \in \Phi_{c}$ if $\varphi$ is independent of the space variable $x$.

If $\varphi \in \Phi_{c}(\Omega)$, we note that there exists its right-derivative $\varphi^{\prime}=\varphi^{\prime}(x, t)$, which is nondecreasing and right continuous, satisfying

$$
\varphi(x, t)=\int_{0}^{t} \varphi^{\prime}(x, s) d s .
$$

This derivative is also denoted by $\varphi_{t}$.

Now let us consider $\varphi \in \Phi_{w}(\Omega)$ and $\gamma>0$. By $\varphi^{-1}(x, \cdot):[0, \infty) \rightarrow[0, \infty]$ we denote the left-continuous inverse of $\varphi$ defined by

$$
\varphi^{-1}(x, t):=\inf \{\tau \geqslant 0: \varphi(x, \tau) \geqslant t\} .
$$

We define some conditions on $\varphi$ which are related to regularity properties with respect to the $x$-variable and the $t$-variable. We say that $\varphi$ satisfies

(A0) if there exists $L \geqslant 1$ such that $\frac{1}{L} \leqslant \varphi^{-1}(x, 1) \leqslant L$ for every $x \in \Omega$.

(A1) if there exists $L \geqslant 1$ such that for any ball $B_{r} \Subset \Omega$ with $\left|B_{r}\right|<1$,

$$
\varphi_{B_{r}}^{+}(t) \leqslant L \varphi_{B_{r}}^{-}(t) \quad \text { for all } t>0 \quad \text { with } \quad \varphi_{B_{r}}^{-}(t) \in\left[1, \frac{1}{\left|B_{r}\right|}\right] \text {. }
$$


(wVA1) if for any $\epsilon>0$, there exists a non-decreasing continuous function $\omega=\omega_{\epsilon}$ : $[0, \infty) \rightarrow[0,1]$ with $\omega(0)=0$ such that for any small ball $B_{r} \Subset \Omega$,

$$
\varphi_{B_{r}}^{+}(t) \leqslant(1+\omega(r)) \varphi_{B_{r}}^{-}(t)+\omega(r) \quad \text { for all } \quad t>0 \quad \text { with } \quad \varphi_{B_{r}}^{-}(t) \in\left[\omega(r), \frac{1}{\left|B_{r}\right|^{1-\epsilon}}\right] .
$$

(aInc) $)_{\gamma}$ if $t \mapsto \frac{\varphi(x, t)}{t^{\gamma}}$ is almost increasing with constant $L \geqslant 1$ uniformly in $x \in \Omega$.

(Inc) $)_{\gamma}$ if $t \mapsto \frac{\varphi(x, t)}{t^{\gamma}}$ is non-decreasing for every $x \in \Omega$.

(aDec $)_{\gamma}$ if $t \mapsto \frac{\varphi(x, t)}{t^{\gamma}}$ is almost decreasing with constant $L \geqslant 1$ uniformly in $x \in \Omega$.

$(\mathrm{Dec})_{\gamma}$ if $t \mapsto \frac{\varphi(x, t)}{t^{\gamma}}$ is non-increasing for every $x \in \Omega$.

We would like to explain these assumptions more informally. Firstly, (A0) condition places us in an "unweighted" space, that is, $\varphi$ is not singular or degenerate with respect to the spatial variable. Secondly, conditions (A1) and (wVA1) are regularity conditions with respect to the spatial variable. The former one is a jump condition, where as the latter is a refined continuity condition. In many cases $\varepsilon$ could be equal to 0 , and this would imply regularity for many special cases such as variable exponent and double phase. However, the weak form catches interesting borderline assumptions for example in double phase case. Last four conditions control the growth of the $\Phi$-function with respect to the $t$-variable. Often we want $\gamma$ in (aInc) $)_{\gamma}$ to be strictly greater than 1 to exclude $L^{1}$ case and finite in $(\mathrm{aDec})_{\gamma}$ to exclude $L^{\infty}$ case. The "almost" part is more flexible and is invariant under equivalent $\Phi$-functions (see the next paragraph). However it allows for local exceptions in growth rate, which (Inc) $\gamma$ and $(\mathrm{Dec})_{\gamma}$ exclude. We also note that $(\mathrm{aDec})_{\gamma}$ implies doubling $\varphi(x, 2 t) \leqslant c \varphi(x, t)$.

The notation $f_{1} \lesssim f_{2}$ means that there exists a constant $C>0$ such that $f_{1} \leqslant C f_{2}$. The notation $f_{1} \approx f_{2}$ means that $f_{1} \lesssim f_{2} \lesssim f_{1}$ whereas $f_{1} \simeq f_{2}$ means that $f_{1}(t / C) \leqslant f_{2}(t) \leqslant$ $f_{1}(C t)$ for some constant $C \geqslant 1$. Throughout this paper, we use these notations when the relevant constants $C$ depend on $n$ and constants in our conditions such as (aInc) $)_{\gamma}$, (Inc) $)_{\gamma}$, $(\mathrm{aDec})_{\gamma},(\mathrm{Dec})_{\gamma}$, and (A0).

Generalized Orlicz space. Let $\varphi \in \Phi_{w}(\Omega)$ and $L^{0}(\Omega)$ be the set of the measurable functions on $\Omega$. The generalized Orlicz space is defined as the set

$$
L^{\varphi}(\Omega):=\left\{f \in L^{0}(\Omega):\|f\|_{L^{\varphi}(\Omega)} \leqslant \infty\right\}
$$

with the (Luxemburg) norm

$$
\|f\|_{L^{\varphi}(\Omega)}:=\inf \left\{\lambda>0: \varrho_{L^{\varphi}}\left(\frac{f}{\lambda}\right) \leqslant 1\right\}
$$

where $\varrho_{\varphi}(g)$ is the modular of $g \in L^{0}(\Omega)$ defined by

$$
\varrho_{L^{\varphi}}(g):=\int_{\Omega} \varphi(x,|g(x)|) d x .
$$

A function $f \in L^{\varphi}(\Omega)$ belongs to the Orlicz-Sobolev space $W^{1, \varphi}(\Omega)$ if its weak partial derivatives $\partial_{1} f, \ldots \partial_{n} f$ exist and belong to $L^{\varphi}(\Omega)$ with the norm

$$
\|f\|_{W^{1, \varphi}(\Omega)}:=\|f\|_{L^{\varphi}(\Omega)}+\sum_{i}\left\|\partial_{i} f\right\|_{L^{\varphi}(\Omega)} .
$$

Furthermore, we denote by $W_{0}^{1, \varphi}(\Omega)$ the closure of $C_{0}^{\infty}(\Omega)$ in $W^{1, \varphi}(\Omega)$. 
Main results. Let $\Omega$ be a bounded open set in $\mathbb{R}^{n}$. Recall that a function $u \in W_{\text {loc }}^{1, \varphi}(\Omega)$ is called a (local) minimizer of the functional

$$
\int_{\Omega} \varphi(x,|\nabla u|) d x
$$

if for every open $\Omega^{\prime} \Subset \Omega$ and every $v \in W^{1, \varphi}\left(\Omega^{\prime}\right)$ with $u-v \in W_{0}^{1, \varphi}\left(\Omega^{\prime}\right)$ we have

$$
\int_{\Omega^{\prime}} \varphi(x,|\nabla u|) d x \leqslant \int_{\Omega^{\prime}} \varphi(x,|\nabla v|) d x .
$$

For a function $\psi: \Omega \rightarrow[-\infty, \infty)$ called the obstacle, the class of admissible functions is defined by

$$
\mathcal{K}_{\psi}^{\varphi}(\Omega):=\left\{u \in W^{1, \varphi}(\Omega) \mid u \geqslant \psi \text { a.e. in } \Omega\right\} .
$$

We define the related solution of the obstacle problem as follows.

Definition 2.3. We say that a function $u \in \mathcal{K}_{\psi}^{\varphi}(\Omega)$ is a (local) minimizer of (2.2) in $\mathcal{K}_{\psi}^{\varphi}(\Omega)$ if it satisfies

$$
\int_{\Omega^{\prime}} \varphi(x,|\nabla u|) d x \leqslant \int_{\Omega^{\prime}} \varphi(x,|\nabla w|) d x
$$

where $w \in \mathcal{K}_{\psi}^{\varphi}(\Omega)$ and $\Omega^{\prime} \Subset \Omega$. If $\varphi \in \Phi_{w}(\Omega) \cap C^{1}([0, \infty))$, we say that a function $u \in \mathcal{K}_{\psi}^{\varphi}(\Omega)$ is a solution to the $\mathcal{K}_{\psi}^{\varphi}(\Omega)$-obstacle problem if it satisfies

$$
\int_{\Omega}\left(\frac{\partial_{t} \varphi(x,|\nabla u|)}{|\nabla u|} \nabla u\right) \cdot \nabla(\eta-u) d x \geqslant 0
$$

for all $\eta \in \mathcal{K}_{\psi}^{\varphi}(\Omega)$ with $\operatorname{supp}(\eta-u) \subset \Omega$, which is equivalent to

$$
\int_{\Omega}\left(\frac{\partial_{t} \varphi(x,|\nabla u|)}{|\nabla u|} \nabla u\right) \cdot \nabla \eta d x \geqslant 0
$$

for all $\eta \in W^{1, \varphi}(\Omega)$ with a compact support and $\eta \geqslant \psi-u$ a.e. in $\Omega$.

Remark 2.5. We note that a solution is also a minimizer and the reverse implication requires differentiability for the $\Phi$-function. More precisely, in the case where $\varphi(x, t)$ is differentiable with respect to $t$ and satisfies $(\mathrm{aDec})$, then solutions and minimizers are equivalent, see [31, Theorem 7.6].

For simplicity, let us define $\partial \varphi=\partial \varphi(x, t): \Omega \times \mathbb{R}^{n} \rightarrow \mathbb{R}^{n}$ by

$$
\partial \varphi(x, t)=\frac{\partial_{t} \varphi(x,|t|)}{|t|} t .
$$

We note that for $g \in W^{1, \varphi}(\Omega)$ with $g \geqslant \psi$ on $\partial \Omega$, the minimizer of the functional

$$
u \in\left\{w \in \mathcal{K}_{\psi}^{\varphi}(\Omega): w=g \text { on } \partial \Omega\right\} \mapsto \int_{\Omega} \varphi(x,|\nabla u|) d x
$$

is the solution to the obstacle problem of $\mathcal{K}_{\psi}^{\varphi}(\Omega)$ with $u=g$ on $\partial \Omega$.

Our first result concerns the local Hölder continuity of the solution. This result does not assume differentiability of our $\Phi$-function or (wVA1). Instead, (A1) condition is enough combined with local Hölder continuity of the obstacle function.

Theorem 2.6. Let $\Omega \subset \mathbb{R}^{n}$ be a bounded domain and $\varphi \in \Phi_{w}(\Omega)$ satisfy (aInc), (aDec), (A0), and (A1). Let $u$ be a minimizer of (2.2) in $\mathcal{K}_{\psi}^{\varphi}(\Omega)$. Suppose that the obstacle $\psi \in$ $C_{\mathrm{loc}}^{0, \beta}(\Omega)$ for some $\beta \in(0,1)$. Then $u \in C_{\mathrm{loc}}^{0, \alpha}(\Omega)$ for some $\alpha \in(0,1)$. 
The proof is based on constructing classical Harnack's inequality. First, a supremum estimate of the solution is proved via a use of Caccioppoli type energy estimate and results in [33]. Compared to previous work, we need to take care of an integral average term to match the supremum estimate with the corresponding infimum estimate.

The following second theorem studies maximal regularity of the solution. Here we need to strengthen our assumptions to include $C^{1}$-regularity of $\varphi$ and the obstacle $\psi$ and replace the assumption (A1) with (wVA1). $C^{1, \alpha}$-regularity requires also a standard decay estimate for modulus of continuity.

Theorem 2.7. Let $\Omega \subset \mathbb{R}^{n}$ be a bounded domain, $\varphi \in \Phi_{w}(\Omega)$ and $\varphi(x, \cdot) \in C^{1}([0, \infty))$ for any $x \in \Omega$ with $\partial_{t} \varphi$ satisfying (A0), (Inc) $)_{p-1}$, (Dec) $)_{q-1}$ for some $1<p \leqslant q$. Let $u \in \mathcal{K}_{\psi}^{\varphi}(\Omega)$ be a solution to the $\mathcal{K}_{\psi}^{\varphi}(\Omega)$-obstacle problem and suppose $\psi \in C_{\text {loc }}^{1, \beta}(\Omega)$ for some $\beta \in(0,1)$.

(i) If $\varphi$ satisfies (wVA1), then $u \in C_{\mathrm{loc}}^{0, \alpha}(\Omega)$ for any $\alpha \in(0,1)$.

(ii) If $\varphi$ satisfies (wVA1) with

$$
\omega(r) \lesssim r^{\delta} \text { for all } r \in(0,1] \text { and for some } \delta>0,
$$

then $u \in C_{\mathrm{loc}}^{1, \alpha}(\Omega)$ for some $\alpha \in(0,1)$.

For higher regularity, the modulus of continuity with respect to $x$ is expected to have vanishing property already in the case of Laplace equation. In addition, continuity of the gradient also requires power-type decay estimate for classical equations, see [27].

For proving results in Theorem 2.7, we first obtain the higher integrability of the gradient of solutions to the obstacle problem which implies the reverse Hölder type inequality. Taking into account the regularized Orlicz function $\tilde{\varphi}$, which was constructed by [36], we derive comparison estimates for the gradients of solutions to the $\mathcal{K}_{\psi}^{\tilde{\varphi}}(\Omega)$-obstacle problem and to $\tilde{\varphi}$-Laplacian equations. The proofs conclude with classical iteration arguments.

Inequalities for generalized Orlicz functions. Below introduce some pointwise inequalities for generalized Orlicz functions. In generalized Orlicz spaces, the classical Hölder conjugate exponent is replaced by a conjugate $\Phi$-function

$$
\varphi^{*}(x, t):=\sup _{s \geqslant 0}(t s-\varphi(x, s)) .
$$

From the definition it is immediate that the generalized Young's inequality

$$
s t \leqslant \varphi(x, s)+\varphi^{*}(x, t)
$$

holds for all $s, t \geqslant 0$.

The following lemmas deal with derivatives of $\Phi$-functions and their proofs can be found in [36, Proposition 3.8].

Lemma 2.10. Let $\varphi \in \Phi_{c} \cap C^{1}([0, \infty))$ with $\varphi^{\prime}$ satisfying $(\mathrm{Inc})_{p-1}$ and (Dec) $)_{q-1}$ for some $1<p \leqslant q$. Then for $\kappa \in(0, \infty)$ and $x, y \in \mathbb{R}^{n}$, the following are satisfied:

(1) $t \varphi^{\prime}(t) \approx \varphi(t)$ and $\varphi$ satisfies (Inc) $)_{p}$ and (Dec) $)_{q}$;

(2) $\frac{\varphi^{\prime}(|x|+|y|)}{|x|+|y|}|x-y|^{2} \approx\left(\frac{\varphi^{\prime}(|x|)}{|x|} x-\frac{\varphi^{\prime}(|y|)}{|y|} y\right) \cdot(x-y)$;

(3) $\frac{\varphi^{\prime}(|x|+|y|)}{|x|+|y|}|x-y|^{2} \lesssim \varphi(|x|)-\varphi(|y|)-\frac{\varphi^{\prime}(|y|)}{|y|} y \cdot(x-y)$;

(4) $\varphi(|x-y|) \lesssim \kappa[\varphi(|x|)+\varphi(|y|)]+\kappa^{-1} \frac{\varphi^{\prime}(|x|+|y|)}{|x|+|y|}|x-y|^{2}$. 
Moreover, if $\varphi \in C^{2}((0, \infty))$, then $t \varphi^{\prime \prime}(t) \approx \varphi^{\prime}(t)$ and $\frac{\varphi^{\prime}(|x|+|y|)}{|x|+|y|}$ can be replaced by $\varphi^{\prime \prime}(|x|+$ $|y|)$ in (2)-(4).

Lemma 2.11 (Propositions 3.5 \& 3.6 [36]). Let $\varphi$ be a $\Phi$-prefunction.

(1) If $\varphi$ satisfies (aInc) $)_{1}$, then there exists $\eta \in \Phi_{c}(\Omega)$ such that $\varphi \simeq \eta$.

(2) If $\varphi$ satisfies (aDec) $)_{1}$, then there exists $\eta \in \Phi_{c}(\Omega)$ such that $\varphi \approx \eta^{-1}$. Note that $\eta^{-1}(x, \cdot)$ is concave.

(3) Let $p, q \in(1, \infty)$. Then $\varphi$ satisfies $(\mathrm{aInc})_{p}$ or $(\mathrm{aDec})_{q}$ if and only if $\varphi^{*}$ satisfies (aDec) $\frac{p}{p-1}$ or $(\mathrm{aInc}) \frac{q}{q-1}$, respectively.

(4) If $\varphi$ satisfies $(\mathrm{aInc})_{p}$ and $(\mathrm{aDec})_{q}$, then for any $s, t \geqslant 0$ and $\kappa \in(0,1)$,

$$
t s \leqslant \varphi\left(x, \kappa^{1 / p} t\right)+\varphi^{*}\left(x, \kappa^{-1 / p} s\right) \lesssim \kappa \varphi(x, t)+\kappa^{\frac{-1}{p-1}} \varphi^{*}(x, s)
$$

and

$$
t s \leqslant \varphi\left(x, \kappa^{1 / q^{\prime}} t\right)+\varphi^{*}\left(x, \kappa^{-1 / q^{\prime}} s\right) \lesssim \kappa^{-(q-1)} \varphi(x, t)+\kappa \varphi^{*}(x, s) .
$$

(5) If $\varphi \in \Phi_{c}(\Omega)$ and $\varphi^{\prime}$ satisfies $(\mathrm{aDec})_{q}$ then

$$
\varphi^{*}\left(x, \frac{\varphi(x, t)}{t}\right) \leqslant \varphi^{*}\left(x, \varphi^{\prime}(x, t)\right) \leqslant t \varphi^{\prime}(t) \approx \varphi(t) .
$$

Lastly we have a Sobolev-Poincaré inequality which is used for higher integrability results.

Lemma 2.12 (Sobolev-Poincaré inequality, Theorem 3.3 [33]). Let $Q_{r} \subset \mathbb{R}^{n}$ be a cube or a ball with diameter $2 r$. Let $\varphi \in \Phi_{w}\left(Q_{r}\right)$ satisfy $(\mathrm{aInc})_{p},(\mathrm{aDec})_{q},(\mathrm{~A} 0)$, and (A1). Let $s<\frac{n}{n-1}$. Then there exists $\beta=\beta(n, s, \varphi)>0$ such that

$$
\left(f_{Q_{r}} \varphi\left(x, \frac{\beta|u|}{r}\right)^{s} d x\right)^{\frac{1}{s}} \lesssim f_{Q_{r}} \varphi(x,|\nabla u|) d x+\frac{\left|\{\nabla u \neq 0\} \cap Q_{r}\right|}{\left|Q_{r}\right|}
$$

for any $u \in W_{0}^{1,1}\left(Q_{r}\right)$ with $\|\nabla u\|_{L^{\varphi}}<1$. If additionally $s \leqslant p$, then

$$
f_{Q_{r}} \varphi\left(x, \beta \frac{\left|u-u_{Q_{r}}\right|}{r}\right) d x \lesssim\left(f_{Q_{r}} \varphi(x,|\nabla u|)^{\frac{1}{s}} d x\right)^{s}+1
$$

for any $u \in W^{1,1}\left(Q_{r}\right)$ with $\|\nabla u\|_{L^{\varphi^{1 / s}}} \leqslant M$, and the implicit constant depends on $M$. The average $u_{Q_{r}}$ can be replaced by $u_{Q}$ for some cube or ball $Q \subset Q_{r}$ with $|Q|>\mu\left|Q_{r}\right|$, in which case the constant depends also on $\mu$.

Essential supremum and infimum estimates. Our first main theorem proven in Section 3 is local Hölder continuity of a solution to an obstacle problem with Hölder continuous obstacle $\psi$. This follows the classical route of estimating supremum and infimum of minimizer $u$ with its integral averages. We prove the essential supremum result in the context of solutions to highlight that the result follows also in that case. The only difference this makes is in the proof of Caccioppoli type inequality in Proposition 2.19 and the requirement for the $\Phi$ function to be differentiable with respect to the second variable. For minimizers, the proof can be modified from an analogous result in [33].

For the following we write

$$
A(k, r):=Q_{r} \cap\{u>k\} .
$$

We first recall the supremum bounds for the local minimizer $u$ of the $\varphi$-energy. 
Proposition 2.15 (Proposition 5.5 and Corollary 5.8 [33]). Let $\varphi \in \Phi_{w}(\Omega)$ satisfy (aInc) , $(\mathrm{aDec})_{q}$, (A0), and (A1). Suppose that $u \in W_{\text {loc }}^{1, \varphi}(\Omega)$ satisfies $\varrho_{L^{\varphi}\left(Q_{2 r}\right)}(\nabla u) \leqslant 1$ for $Q_{2 r} \subset \Omega$. Suppose that u satisfies the Caccioppoli inequality

$$
\int_{A(\ell, r)} \varphi\left(x,\left|\nabla(u-\ell)_{+}\right|\right) d x \lesssim \int_{A(\ell, 2 r)} \varphi\left(x, \frac{(u-\ell)_{+}}{r}\right) d x
$$

for any $\ell \geqslant 0$. Then $u_{+}$is bounded and

$$
\underset{Q_{r / 2}}{\operatorname{ess} \sup } u_{+} \lesssim\left(f_{Q_{r}} u_{+}^{q} d x\right)^{\frac{1}{q}}+\left|u_{Q_{r / 2}}\right|+r
$$

for any $Q_{r} \subset \Omega$. The term $\left|u_{Q_{r / 2}}\right|$ can be omitted if $u$ is non-negative.

Furthermore, if $u \in L^{\infty}\left(Q_{r}\right)$ satisfies (2.17) without the term $\left|u_{Q_{r / 2}}\right|$, then

$$
\underset{Q_{r / 2}}{\operatorname{essip}} u_{+} \lesssim\left(f_{Q_{r}} u_{+}^{h} d x\right)^{\frac{1}{h}}+r
$$

for any $h \in(0, \infty)$.

We need similar supremum and infimum bounds for the solutions of the obstacle problem. We start with the supremum estimate and base our proof on [33, Section 5]. Therefore we only need to prove that solution to the obstacle problem satisfies the Caccioppoli type energy estimate (2.16). Note that in the case of obstacle problems, we need to restrict possible values of $\ell$ using the obstacle $\psi$.

Proposition 2.19. Let $\varphi \in \Phi_{w}(\Omega) \cap C^{1}([0, \infty))$ satisfy (aInc) $)_{p}$, (aDec $)_{q}$, (A0), and (A1). Let $u \in \mathcal{K}_{\psi}^{\varphi}(\Omega)$ be a solution to the $\mathcal{K}_{\psi}^{\varphi}(\Omega)$ - obstacle problem. Then if $\psi \in W^{1, \varphi}(\Omega) \cap L_{\mathrm{loc}}^{\infty}(\Omega)$ and $\theta \in\left[\frac{1}{2}, 1\right)$ we have

$$
\underset{Q_{\theta r}}{\operatorname{ess} \sup }(u-\ell)_{+} \lesssim(1-\theta)^{-4 n q^{2}}\left[\left(f_{Q_{r}}(u-\ell)_{+}^{q} d x\right)^{1 / q}+\left|(u-\ell)_{Q_{r / 2}}\right|\right]+r
$$

for any $Q_{2 r} \subset \Omega$ and $\ell \geqslant \sup _{Q_{2 r}} \psi$, provided that $\varrho_{L^{\varphi}\left(Q_{2 r}\right)}(|\nabla u|) \leqslant 1$. The term $\left|(u-\ell)_{Q_{r / 2}}\right|$ can be omitted if $u-\ell$ is non-negative almost everywhere in $Q_{2 r}$.

Furthermore, if $u \in L^{\infty}\left(Q_{r}\right)$ satisfies (2.20) without the term $\left|(u-\ell)_{Q_{r / 2}}\right|$, then

$$
\underset{Q_{r / 2}}{\operatorname{ess} \sup }(u-\ell)_{+} \lesssim\left(f_{Q_{r}}(u-\ell)_{+}^{h} d x\right)^{\frac{1}{h}}+r
$$

for any $h \in(0, \infty)$.

Proof. If $v:=u-\ell$ satisfies Caccioppoli inequality (2.16), then Proposition 2.15 implies the desired bound. Hence it suffices to show that $v$ satisfies (2.16) for $\ell \geqslant \sup _{Q_{2 r}} \psi$.

Consider any cubes $Q_{\sigma} \subset Q_{\varrho} \subset Q_{2 r}$. Let $k \geqslant 0$ and let $\tau \in C_{0}^{\infty}\left(Q_{\varrho}\right)$ be a cut off function such that $0 \leqslant \tau \leqslant 1$ in $Q_{\varrho}, \tau=1$ in $Q_{\sigma}$ and $|\nabla \tau| \leqslant \frac{c(n)}{\varrho-\sigma}$.

Since $(v-k)_{+}=(u-\ell-k)_{+} \leqslant u-\psi$, we take a test function $\eta=-(v-k)_{+} \tau^{q}$ in (2.4) to discover that

$$
\begin{aligned}
& \int_{A(k+\ell, \varrho)} \partial \varphi(x,|\nabla u|) \cdot \nabla\left(-(v-k)_{+} \tau^{q}\right) d x \\
& =-\int_{A(k+\ell, \varrho)}[\partial \varphi(x,|\nabla u|) \cdot \nabla u] \tau^{q} d x-q \int_{A(k+\ell, \varrho)}[\partial \varphi(x,|\nabla u|) \cdot \nabla \tau](v-k)_{+} \tau^{q-1} d x \geqslant 0,
\end{aligned}
$$


where $A(k+\ell, \varrho):=Q_{\varrho} \cap\{u>\ell+k\}$. Then from Lemma 2.11 (5) and the fact that $\varphi^{*}\left(x, \varphi_{t}(x, t)\right) \approx \varphi^{*}(x, \varphi(x, t) / t) \approx \varphi(x, t)$ and $\varphi^{*}$ satisfies (aInc) $\frac{q}{q-1}$, we deduce with (aDec) that

$$
\begin{aligned}
& \int_{A(k+\ell, \varrho)} \varphi(x,|\nabla u|) \tau^{q} d x \lesssim \int_{A(k+\ell, \varrho)} \partial \varphi(x,|\nabla u|)|\nabla u| \tau^{q} d x \\
& \quad \leqslant q \int_{A(k+\ell, \varrho)}|\partial \varphi(x,|\nabla u|)||\nabla \tau|(v-k)_{+} \tau^{q-1} d x \\
& \quad \lesssim \kappa \int_{A(k+\ell, \varrho)} \varphi^{*}\left(x,|\partial \varphi(x,|\nabla u|)| \tau^{q-1}\right) d x+\frac{1}{\kappa^{q-1}} \int_{Q_{\varrho}} \varphi\left(x,|\nabla \tau|(v-k)_{+}\right) d x \\
& \quad \lesssim \kappa \int_{A(k+\ell, \varrho)} \varphi^{*}(x,|\partial \varphi(x,|\nabla u|)|) \tau^{q} d x+\frac{1}{\kappa^{q-1}} \int_{Q_{\varrho}} \varphi\left(x,|\nabla \tau|(v-k)_{+}\right) d x \\
& \quad \lesssim \kappa \int_{A(k+\ell, \varrho)} \varphi(x,|\nabla u|) \tau^{q} d x+c_{\kappa} \int_{Q_{\varrho}} \varphi\left(x, \frac{(v-k)_{+}}{\varrho-\sigma}\right) d x
\end{aligned}
$$

for any $\kappa \in(0,1)$. Therefore, by choosing $\kappa$ sufficiently small, we conclude

$$
\int_{Q_{\sigma}} \varphi\left(x,\left|\nabla(v-k)_{+}\right|\right) \tau^{q} d x \lesssim \int_{Q_{\varrho}} \varphi\left(x, \frac{(v-k)_{+}}{\varrho-\sigma}\right) d x .
$$

Since solution to an obstacle problem is also a superminimizer, the standard arguments provide the following weak Harnack inequality, see [33, Corollary 6.4].

Proposition 2.22. Let $\varphi \in \Phi_{w}(\Omega)$ satisfy $(\mathrm{aInc})_{p}$, $(\mathrm{aDec})_{q}$, (A0), and (A1). Suppose that $u \in W_{\text {loc }}^{1, \varphi}(\Omega)$ is a non-negative solution of the $\mathcal{K}_{\psi}^{\varphi}(\Omega)$-obstacle problem. Then there exists $h_{0}>0$ such that

$$
\left(f_{Q_{r}} u^{h_{0}} d x\right)^{\frac{1}{h_{0}}} \lesssim \underset{Q_{r / 2}}{\operatorname{essinf}} u+r
$$

when $Q_{2 r} \subset \Omega$ and $\varrho_{L^{\varphi}\left(Q_{2 r}\right)}(|\nabla u|) \leqslant 1$.

\section{HÖLDER CONTINUITY}

Now we are ready to prove local Hölder continuity of the solution to an obstacle problem with Hölder continuous obstacle. Compared to higher regularity results in later sections, we assume the weaker condition (A1) instead of (wVA1).

Proof of Theorem 2.6. Since $\psi \in C_{\mathrm{loc}}^{0, \beta}(\Omega)$ for some $\beta \in(0,1)$, we note that for any $Q_{2 r} \subset \Omega$ with $r<1$, there exists a constant $[\psi]_{\beta}>0$ such that

$$
|\psi(x)-\psi(y)| \leqslant[\psi]_{\beta}|x-y|^{\beta} \text { for all } x, y \in Q_{2 r} .
$$

From Propositions 2.19-2.22, we note that $u$ is locally bounded in $\Omega$. Then for $Q_{2 r} \subset \Omega$, we define

$$
\bar{u}(r):=\operatorname{ess~sup}_{x \in Q_{r}} u(x), \quad \underline{u}(r):=\operatorname{essinf}_{x \in Q_{r}} u(x), \quad \bar{\psi}(r):=\operatorname{ess~sup}_{x \in Q_{r}} \psi(x), \underline{\psi}(r):=\operatorname{essinf}_{x \in Q_{r}}^{\operatorname{ess}} \psi(x) .
$$

Next we consider two cases: $\underline{u}(r) \geqslant \bar{\psi}(r)$ and $\underline{u}(r) \leqslant \bar{\psi}(r)$. For the first case we can use Proposition 2.19 with $\ell=\underline{u}(r)$. As $u-\underline{u}(r)$ is always nonnegative in $Q_{r}$, we can omit the 
average term and this yields an equivalent form of (2.21)

$$
\bar{u}(r / 4)-\underline{u}(r)=\operatorname{ess}_{Q_{r / 4}}^{\sup }(u-\underline{u}(r)) \lesssim\left[f_{Q_{r / 2}}(u-\underline{u}(r))^{h} d x\right]^{1 / h}+r
$$

for any $h \in(0, \infty)$.

For the second case, applying Proposition 2.19 with $\ell=\bar{\psi}(r)$ and local Hölder continuity of $\psi$, we get

$$
\begin{aligned}
& \underset{Q_{\theta r / 2}}{\operatorname{ess} \sup }(u-\underline{u}(r)) \leqslant \underset{Q_{\theta r / 2}}{\operatorname{ess} \sup }(u-\underline{\psi}(r))_{+} \leqslant \operatorname{ess\operatorname {sup}}(u-\bar{\psi}(r))_{+}+[\psi]_{\beta} r^{\beta} \\
& \lesssim(1-\theta)^{-4 n q^{2}}\left[\left(f_{Q_{r / 2}}(u-\bar{\psi}(r))_{+}^{q} d x\right)^{1 / q}+\left|(u-\bar{\psi}(r))_{Q_{r / 2}}\right|\right]+r+r^{\beta} \\
& \lesssim(1-\theta)^{-4 n q^{2}}\left[\left(f_{Q_{r / 2}}(u-\underline{u}(r))_{+}^{q} d x\right)^{1 / q}+\left|(u-\bar{\psi}(r))_{Q_{r / 2}}\right|\right]+r^{\beta} .
\end{aligned}
$$

Let us briefly focus on the average term. Again, using the Hölder continuity of the obstacle and the inequality $\underline{u}(r) \leqslant \bar{\psi}(r)$ combined with Hölder inequality to increase the exponent we get

$$
\begin{aligned}
\left|(u-\bar{\psi}(r))_{Q_{r / 2}}\right| & =\left|f_{Q_{r / 2}} u-\bar{\psi}(r) d x\right|=\left|f_{Q_{r / 2}} u-\underline{\psi}(r)+\underline{\psi}(r)-\bar{\psi}(r) d x\right| \\
& \leqslant\left|f_{Q_{r / 2}} u-\underline{\psi}(r) d x\right|+|\underline{\psi}(r)-\bar{\psi}(r)| \leqslant f_{Q_{r / 2}} u-\underline{\psi}(r) d x+[\psi]_{\beta} r^{\beta} \\
& \leqslant f_{Q_{r / 2}}(u-\bar{\psi}(r))_{+} d x+2[\psi]_{\beta} r^{\beta} \leqslant f_{Q_{r / 2}} u-\underline{u}(r) d x+2[\psi]_{\beta} r^{\beta} \\
& \leqslant\left[f_{Q_{r / 2}}(u-\underline{u}(r))^{q} d x\right]^{1 / q}+2[\psi]_{\beta} r^{\beta} .
\end{aligned}
$$

Combining the two previous estimates we have

$$
\underset{Q_{\theta r / 2}}{\operatorname{ess} \sup }(u-\underline{u}(r)) \lesssim(1-\theta)^{-4 n q^{2}}\left[f_{Q_{r / 2}}(u-\underline{u}(r))^{q} d x\right]^{1 / q}+r^{\beta} .
$$

Therefore, performing the iteration argument in the same way as the proof of [33, Corollary 5.8], we obtain

$$
\underset{Q_{r / 4}}{\operatorname{ess} \sup }(u-\underline{u}(r)) \lesssim\left[f_{Q_{r / 2}}(u-\underline{u}(r))^{h} d x\right]^{1 / h}+r^{\beta}
$$

for any $h \in(0, \infty)$.

Combining the inequalities (3.1)-(3.2) and choosing $h=h_{0}$ where $h_{0}$ is given in Proposition 2.22 we get

$$
\bar{u}(r / 4)-\underline{u}(r) \lesssim\left[f_{Q_{r / 2}}(u-\underline{u}(r))^{h_{0}} d x\right]^{1 / h_{0}}+r^{\beta}
$$


Since for a fixed $r$, the function $u-\underline{u}(r)$ is a nonnegative solution to the $\mathcal{K}_{\psi-\underline{u}(r)}^{\varphi}$-obstacle problem, Proposition 2.22 yields that

$$
\left[f_{Q_{r / 2}}(u-\underline{u}(r))^{h_{0}} d x\right]^{1 / h_{0}} \lesssim \underline{u}(r / 4)-\underline{u}(r)+r .
$$

Combining this with (3.3) we arrive at

$$
\bar{u}(r / 4)-\underline{u}(r) \leqslant C(\underline{u}(r / 4)-\underline{u}(r))+c r^{\beta}
$$

for some constants $C, c>0$. Again there are two cases. First if $(C+1)(\underline{u}(r / 4)-\underline{u}(r)) \leqslant$ $\bar{u}(r)-\underline{u}(r)$, then

$$
\bar{u}(r / 4)-\underline{u}(r / 4) \leqslant \bar{u}(r / 4)-\underline{u}(r) \leqslant \frac{C}{C+1}(\bar{u}(r)-\underline{u}(r))+c r^{\beta} .
$$

On the other hand, if $(C+1)(\underline{u}(r / 4)-\underline{u}(r))>\bar{u}(r)-\underline{u}(r)$, then

$$
\begin{aligned}
\bar{u}(r / 4)-\underline{u}(r / 4) & =\bar{u}(r / 4)-\underline{u}(r)-(\underline{u}(r / 4)-\underline{u}(r)) \\
& <\bar{u}(r / 4)-\underline{u}(r)-\frac{1}{C+1}(\bar{u}(r)-\underline{u}(r)) \\
& \leqslant \frac{C}{C+1}(\bar{u}(r)-\underline{u}(r)) .
\end{aligned}
$$

From both cases we arrive to a conclusion that

$$
\begin{aligned}
\operatorname{osc}(u, r / 4):=\bar{u}(r / 4)-\underline{u}(r / 4) & \leqslant \frac{C}{C+1}(\bar{u}(r)-\underline{u}(r))+c r^{\beta} \\
& =\frac{C}{C+1} \operatorname{osc}(u, r)+c r^{\beta} .
\end{aligned}
$$

\section{Higher REGULARITY OF THE SOLUTION}

For the rest of the paper we assume that $\psi \in C_{\text {loc }}^{1, \beta}(\Omega)$ for some $\beta \in(0,1)$. Then we note that for any $\Omega^{\prime} \Subset \Omega$, there exists a constant $[\nabla \psi]_{\beta}>0$ such that

$$
|\nabla \psi(x)-\nabla \psi(y)| \leqslant[\nabla \psi]_{\beta}|x-y|^{\beta} \text { for all } x, y \in \Omega^{\prime} .
$$

In the previous section, we proved the first result in Theorem 2.6 with cubes because of the simple proof based on the previous results, especially, supremum and infimum estimates with cubes. However, from now on we use balls instead of cubes in order to prove our second result in Theorem 2.7. It can be also proved with cubes by the same approach we use here, but seems to be more complicated because in the process of its proof it has to mix cubes and balls when dealing with Calderón-Zygmund type estimates.

4.1. Higher integrability of the gradient. We start this section by introducing some higher integrability results of $\nabla u$.

Lemma 4.1 (Higher Integrability, Theorem 1.1 [32]). Let $\varphi \in \Phi_{w}(\Omega)$ satisfy (A0), (A1), $(\mathrm{aInc})_{p}$ and $(\mathrm{aDec})_{q}$ with constant $L \geqslant 1$ and $1<p \leqslant q<\infty$. If $u \in W_{\mathrm{loc}}^{1, \varphi}(\Omega)$ is $a$ local minimizer of the $\varphi$-energy (2.2) then there exist $\sigma_{0}=\sigma_{0}(n, p, q, L)>0$ and $c_{1}=$ $c_{1}(n, p, q, L) \geqslant 1$ such that

$$
\left(f_{B_{r}} \varphi(x,|\nabla u|)^{1+\sigma_{0}} d x\right)^{\frac{1}{1+\sigma_{0}}} \leqslant c_{1}\left(f_{B_{2 r}} \varphi(x,|\nabla u|) d x+1\right)
$$

for any $B_{2 r} \Subset \Omega$ with $\|\nabla u\|_{L^{\varphi}\left(B_{2 r}\right)} \leqslant 1$. 
Lemma 4.3 (Reverse Hölder type inequality, Lemma 4.7 [36]). Assume that $u \in W_{\text {loc }}^{1, \varphi}(\Omega)$ satisfies (4.2) for some $B_{2 r} \Subset \Omega$. For every $t \in(0,1]$ there exists $c=c\left(c_{1}, t, q\right)>0$ such that

$$
\left(f_{B_{r}} \varphi(x,|\nabla u|)^{1+\sigma_{0}} d x\right)^{\frac{1}{1+\sigma_{0}}} \leqslant c\left[\left(f_{B_{2 r}} \varphi(x,|\nabla u|)^{t} d x\right)^{\frac{1}{t}}+1\right] .
$$

Moreover, if $\varphi$ satisfies (A0), (A1) and (aDec) $)_{q}$ with $L \geqslant 1$ and $q>1$, and $\|\nabla u\|_{L^{\varphi}\left(B_{2 r}\right)} \leqslant 1$, then

$$
f_{B_{r}} \varphi(x,|\nabla u|) d x \leqslant\left(f_{B_{r}} \varphi(x,|\nabla u|)^{1+\sigma_{0}} d x\right)^{\frac{1}{1+\sigma_{0}}} \leqslant c\left(\varphi_{B_{2 r}}^{-}\left(f_{B_{2 r}}|\nabla u| d x\right)+1\right)
$$

for some $c=c\left(c_{1}, q, L\right) \geqslant 1$.

The next Lemma generalizes previous results to the obstacle case. The proof is a standard combination of Caccioppoli inequality, Sobolev-Poincaré inequality and Gehring's lemma.

Lemma 4.4. Let $\varphi \in \Phi_{w}(\Omega) \cap C^{1}([0, \infty))$ satisfy (A0), (A1), (aInc) $)_{p}$ and (aDec) $)_{q}$ with constant $L \geqslant 1$ and $1<p \leqslant q<\infty$. Assume that $u$ is a solution to the $\mathcal{K}_{\psi}^{\varphi}(\Omega)$-obstacle problem and $B_{2 r} \Subset \Omega$ with $r>0$ satisfying $\varrho_{L^{\varphi}\left(B_{2 r}\right)}(|\nabla u|), \varrho_{L^{\varphi}\left(B_{2 r}\right)}(|\nabla \psi|) \leqslant 1$. Then there exists $\sigma_{0} \in(0,1)$ such that $\varphi(\cdot,|\nabla u|) \in L^{1+\sigma_{0}}\left(B_{r}\right)$, and for any $\sigma \in\left(0, \sigma_{0}\right]$,

$$
f_{B_{r}} \varphi(x,|\nabla u|)^{1+\sigma} d x \leqslant c_{2}\left[\left(f_{B_{2 r}} \varphi(x,|\nabla u|) d x\right)^{1+\sigma}+f_{B_{2 r}} \varphi(x,|\nabla \psi|)^{1+\sigma} d x+1\right]
$$

for some $c_{2}=c_{2}(n, p, q, L)>0$.

Proof. Let $\tau \in C_{0}^{\infty}\left(B_{2 r}\right)$ be a cut off function such that $0 \leqslant \tau \leqslant 1, \tau \equiv 1$ in $B_{r}$ and $|\nabla \tau| \leqslant$ $\frac{c(n)}{r}$. Since $\psi-\bar{\psi}_{B_{2 r}}-u+\bar{u}_{B_{2 r}} \geqslant \psi-u$, we note that $\eta:=\tau^{q}\left(\psi-\bar{\psi}_{B_{2 r}}-u+\bar{u}_{B_{2 r}}\right) \geqslant \psi-u$ in $B_{2 r}$ where $q$ is given in (aDec $)_{q}$. Then we take $\eta$ as a test function in (2.4) to have that

$$
\int_{B_{2 r}} \partial \varphi(x, \nabla u) \cdot \nabla \eta d x \geqslant 0
$$

which implies that

$$
\begin{aligned}
\int_{B_{2 r}}[\partial \varphi(x, \nabla u) \cdot \nabla u] \tau^{q} d x \leqslant & \int_{B_{2 r}}[\partial \varphi(x, \nabla u) \cdot \nabla \psi] \tau^{q} d x \\
& +q \int_{B_{2 r}}[\partial \varphi(x, \nabla u) \cdot \nabla \tau] \tau^{q-1}\left(\psi-\bar{\psi}_{B_{2 r}}-u+\bar{u}_{B_{2 r}}\right) d x .
\end{aligned}
$$


Then applying Lemma 2.10 (2) and Lemma 2.11 (4)-(5),

$$
\begin{aligned}
& \int_{B_{r}} \varphi(x,|\nabla u|) d x \lesssim \int_{B_{2 r}}[\partial \varphi(x, \nabla u) \cdot \nabla u] \tau^{q} d x \\
& \leqslant \int_{B_{2 r}}[\partial \varphi(x, \nabla u) \cdot \nabla \psi] \tau^{q} d x \\
& \quad+q \int_{B_{2 r}}[\partial \varphi(x, \nabla u) \cdot \nabla \tau] \tau^{q-1}\left(\psi-\bar{\psi}_{B_{2 r}}-u+\bar{u}_{B_{2 r}}\right) d x \\
& \lesssim \kappa \int_{B_{2 r}} \varphi^{*}(x,|\partial \varphi(x, \nabla u)|) d x+c(\kappa) \int_{B_{2 r}} \varphi\left(x,|\nabla \psi| \tau^{q}\right) d x \\
& \quad+q \kappa \int_{B_{2 r}} \varphi^{*}(x,|\partial \varphi(x, \nabla u)|) d x+q c(\kappa) \int_{B_{2 r}} \varphi\left(x, \tau^{q-1}|\nabla \tau|\left|\psi-\bar{\psi}_{B_{2 r}}-u+\bar{u}_{B_{2 r}}\right|\right) d x \\
& \lesssim \kappa \int_{B_{2 r}} \varphi(x,|\nabla u|) d x+\int_{B_{2 r}} \varphi(x,|\nabla \psi|) d x \\
& \quad+\int_{B_{2 r}} \varphi\left(x, \frac{\left|u-\bar{u}_{B_{2 r}}\right|}{r}\right) d x+\int_{B_{2 r}} \varphi\left(x, \frac{\left|\psi-\bar{\psi}_{B_{2 r}}\right|}{r}\right) d x
\end{aligned}
$$

for any $\kappa \in(0,1)$. Therefore, by choosing $\kappa$ sufficiently small, we have

$$
\begin{aligned}
f_{B_{r}} \varphi(x,|\nabla u|) d x \lesssim & f_{B_{2 r}} \varphi\left(x, \frac{\left|u-\bar{u}_{B_{2 r}}\right|}{r}\right) d x \\
& +f_{B_{2 r}} \varphi(x,|\nabla \psi|) d x+f_{B_{2 r}} \varphi\left(x, \frac{\left|\psi-\bar{\psi}_{B_{2 r}}\right|}{r}\right) d x
\end{aligned}
$$

Here, Sobolev-Poincaré inequality (2.14) yields that for $1<s \leqslant p$,

$$
f_{B_{2 r}} \varphi\left(x, \frac{\left|u-\bar{u}_{B_{2 r}}\right|}{r}\right) d x \lesssim\left(f_{B_{2 r}} \varphi(x,|\nabla u|)^{\frac{1}{s}} d x\right)^{s}+1
$$

because

$$
\int_{B_{2 r}} \varphi(x,|\nabla u|)^{\frac{1}{s}} d x \leqslant \int_{B_{2 r}} \varphi(x,|\nabla u|) d x+1 \leqslant 2 .
$$

Similarly, we have that

$$
f_{B_{2 r}} \varphi\left(x, \frac{\left|\psi-\bar{\psi}_{B_{2 r}}\right|}{r}\right) d x \lesssim\left(f_{B_{2 r}} \varphi(x,|\nabla \psi|)^{\frac{1}{s}} d x\right)^{s}+1 \leqslant f_{B_{2 r}} \varphi(x,|\nabla \psi|) d x+1,
$$

because

$$
\int_{B_{2 r}} \varphi(x,|\nabla \psi|)^{\frac{1}{s}} d x \leqslant \int_{B_{2 r}} \varphi(x,|\nabla \psi|) d x+1 \leqslant 2 .
$$

Hence, we conclude that

$$
f_{B_{r}} \varphi(x,|\nabla u|) d x \lesssim\left(f_{B_{2 r}} \varphi(x,|\nabla u|)^{\frac{1}{s}} d x\right)^{s}+f_{B_{2 r}} \varphi(x,|\nabla \psi|) d x+1 .
$$

Since $\nabla \psi \in L_{\text {loc }}^{\infty}(\Omega)$, by Gehring's lemma (see [29, Theorem 6.6 and Corollary 6.1, pp. 203-204]), we obtain the desired estimates, when we denote the implicit constant by $c_{2}$.

We can upgrade the higher integrability for the obstacle problem like in the case for minimizers. 
Lemma 4.6 (Reverse Hölder type inequality). Under the same hypotheses as in Lemma 4.4, for every $t \in(0,1]$, there exists $c_{t}=c_{t}\left(c_{2}, t, q\right)>0$ such that

$$
\begin{aligned}
& \left(f_{B_{r}} \varphi(x,|\nabla u|)^{1+\sigma_{0}} d x\right)^{1 /\left(1+\sigma_{0}\right)} \\
& \quad \leqslant c_{t}\left[\left(f_{B_{2 r}} \varphi(x,|\nabla u|)^{t} d x\right)^{1 / t}+\left(f_{B_{2 r}} \varphi(x,|\nabla \psi|)^{1+\sigma_{0}} d x\right)^{1 /\left(1+\sigma_{0}\right)}+1\right] .
\end{aligned}
$$

Additionally, if $\varphi$ satisfies (A0), (A1), $(\mathrm{aDec})_{q}$ with constant $L \geqslant 1$ and $q>1$, and $\|\nabla u\|_{L^{\varphi}\left(B_{2 r}\right)} \leqslant 1$, then

$$
\begin{aligned}
f_{B_{r}} \varphi(x,|\nabla u|) d x & \leqslant\left(f_{B_{r}} \varphi(x,|\nabla u|)^{1+\sigma_{0}} d x\right)^{1 /\left(1+\sigma_{0}\right)} \\
& \leqslant c\left[\varphi_{B_{2 r}}^{-}\left(f_{B_{2 r}}|\nabla u| d x\right)+\left(f_{B_{2 r}} \varphi(x,|\nabla \psi|)^{1+\sigma_{0}} d x\right)^{1 /\left(1+\sigma_{0}\right)}+1\right],
\end{aligned}
$$

where $c \geqslant 1$ depends on $c_{2}, q$ and $L$.

Proof. The first inequality (4.7) follows from (4.5) by the same argument as in [29, Remark 6.12, pp. 205].

The second inequality (4.8) can be derived by the same way as in the proof of Lemma 4.7 of [36], We choose $t=1 / q$ in the inequality (4.7) to obtain

$$
\begin{aligned}
& \left(f_{B_{r}} \varphi(x,|\nabla u|)^{1+\sigma_{0}} d x\right)^{1 /\left(1+\sigma_{0}\right)} \\
& \lesssim\left(f_{B_{2 r}} \varphi_{B_{2 r}}^{+}(|\nabla u|)^{1 / q} d x\right)^{q}+\left(f_{B_{2 r}} \varphi(x,|\nabla \psi|)^{1+\sigma_{0}} d x\right)^{1 /\left(1+\sigma_{0}\right)}+1 .
\end{aligned}
$$

As $\varphi$ satisfies $(\mathrm{aDec})_{q}$, we immediately see that $\varphi^{+}(t)^{1 / q}$ satisfies $(\mathrm{aDec})_{1}$. Thus using Lemma 2.11 (2) and Jensen's inequality for concave functions we arrive at

$$
\begin{aligned}
& \left(f_{B_{r}} \varphi(x,|\nabla u|)^{1+\sigma_{0}} d x\right)^{1 /\left(1+\sigma_{0}\right)} \\
& \lesssim \varphi_{B_{2 r}}^{+}\left(f_{B_{2 r}}|\nabla u| d x\right)+\left(f_{B_{2 r}} \varphi(x,|\nabla \psi|)^{1+\sigma_{0}} d x\right)^{1 /\left(1+\sigma_{0}\right)}+1 .
\end{aligned}
$$

Now we want to use (A1), for which we need that

$$
\varphi_{B_{2 r}}^{-}\left(f_{B_{2 r}}|\nabla u| d x\right) \leqslant \frac{1}{\left|B_{2 r}\right|},
$$

but this follows from Jensen's inequality since $\varrho_{\varphi_{B_{2 r}}^{-}}(|\nabla u|) \leqslant \varrho_{\varphi}(|\nabla u|) \leqslant 1$ by assumption that $\|\nabla u\|_{L^{\varphi}\left(B_{2 r}\right)} \leqslant 1$. Then if $\varphi_{B_{2 r}}^{-}\left(f_{B_{2 r}}|\nabla u| d x\right)^{2} \geqslant 1$, (A1) yields that

$$
\varphi_{B_{2 r}}^{+}\left(f_{B_{2 r}}|\nabla u| d x\right) \lesssim \varphi_{B_{2 r}}^{-}\left(f_{B_{2 r}}|\nabla u| d x\right) .
$$


In the other case, that is $\varphi_{B_{2 r}}^{-}\left(f_{B_{2 r}}|\nabla u| d x\right)<1$ the constant term dominates in (4.8). Then $\varphi_{B_{2 r}}^{+}\left(f_{B_{2 r}}|\nabla u| d x\right)$ can be estimated by the constant $c$ with the help of (aDec) and (A0).

4.2. Comparison functions. Now we are ready to prove some essential estimates for obtaining higher regularity. The first step is to construct a suitable reference problem introducing a regularized Orlicz function $\tilde{\varphi}$. We use the function $\tilde{\varphi}$ for Orlicz type equations, which are known to have solutions with $C_{\text {loc }}^{1, \alpha}$-regularity.

For the rest of the paper, we assume that $\varphi \in \Phi_{c}(\Omega) \cap C^{1}([0, \infty))$ satisfies (wVA1) with $\varphi^{\prime}$ satisfying (Inc) $)_{p-1}$ and (Dec) $)_{q-1}$ for some $1<p \leqslant q$.

From (wVA1), we note that $\varphi$ satisfies a stronger version of (A1) i.e., there exist $L \geqslant 1$ and a non-decreasing, bounded, continuous function $\omega:[0, \infty) \rightarrow[0,1]$ with $\omega(0)=0$ such that for any small ball $B_{r} \Subset \Omega$

$$
\varphi_{B_{r}}^{+}(t) \leqslant L \varphi_{B_{r}}^{-}(t) \quad \text { for all } \quad t>0 \quad \text { with } \quad \varphi_{B_{r}}^{-}(t) \in\left[\omega(r), \frac{1}{\left|B_{r}\right|}\right] .
$$

We further assume that $\varphi^{\prime}$ satisfies (A0) with the same constant $L \geqslant 1,(\text { Inc })_{p-1}$ and (Dec) $)_{q-1}$ for some $1<p \leqslant q$.

In order to use higher integrability results we need to assume that $|\nabla u|$ and $|\nabla \psi|$ are small in sense of norms. Recall that we assume $\psi$ to have continuous gradient, so the gradient has higher integrability over a compact set. These are achieved by considering as the integration domain a small enough ball. To quantify this smallness we henceforth fix $\Omega^{\prime} \Subset \Omega$ and consider $B_{2 r}=B\left(x_{0}, 2 r\right) \subset \Omega^{\prime}$ with $r>0$ so that

$r \leqslant \frac{1}{2}, \omega(2 r) \leqslant \frac{1}{L}$ and

$\left|B_{2 r}\right| \leqslant \min \left\{\frac{1}{2 L}, 2^{-\frac{2\left(1+\sigma_{0}\right)}{\sigma_{0}}}\left(\int_{\Omega^{\prime}} \varphi(x,|\nabla u|)^{1+\sigma_{0}} d x\right)^{-\frac{2+\sigma_{0}}{\sigma_{0}}}, 2^{-\frac{2\left(1+\sigma_{0}\right)}{\sigma_{0}}}\left(\int_{\Omega^{\prime}} \varphi(x,|\nabla \psi|)^{1+\sigma_{0}} d x\right)^{-\frac{2+\sigma_{0}}{\sigma_{0}}}\right\}$,

where $\sigma_{0} \in(0,1)$ is given in Lemma 4.4. Hence we have from the above assumption (4.10) that

$$
\begin{aligned}
& \int_{B_{2 r}} \varphi(x,|\nabla u|) d x \leqslant \int_{B_{2 r}} \varphi(x,|\nabla u|)^{1+\frac{\sigma_{0}}{2}}+1 d x \\
& \leqslant\left|B_{2 r}\right|\left(f_{B_{2 r}} \varphi(x,|\nabla u|)^{1+\sigma_{0}} d x\right)^{\frac{2+\sigma_{0}}{2\left(1+\sigma_{0}\right)}}+\left|B_{2 r}\right| \leqslant \frac{1}{2}+\frac{1}{2}=1,
\end{aligned}
$$

and similarly,

$$
\begin{aligned}
& \int_{B_{2 r}} \varphi(x,|\nabla \psi|) d x \leqslant \int_{B_{2 r}} \varphi(x,|\nabla \psi|)^{1+\frac{\sigma_{0}}{2}}+1 d x \\
& \leqslant\left|B_{2 r}\right|\left(f_{B_{2 r}} \varphi(x,|\nabla \psi|)^{1+\sigma_{0}} d x\right)^{\frac{2+\sigma_{0}}{2\left(1+\sigma_{0}\right)}}+\left|B_{2 r}\right| \leqslant \frac{1}{2}+\frac{1}{2}=1
\end{aligned}
$$

by applying Hölder's inequality. Then the hypotheses in Lemma 4.4 are satisfied and so it follows that for a solution $u$ to the $\mathcal{K}_{\psi}^{\varphi}(\Omega)$-obstacle problem, $\varphi(\cdot,|\nabla u|) \in L_{l o c}^{1+\sigma_{0}}(\Omega)$ and (4.5) holds.

For comparison argument, we shall use the regularized Orlicz function $\tilde{\varphi}$ constructed from a generalized Orlicz function $\varphi$, which was introduced in [36]. We now give its definition 
and list its properties that will be used in our proof; see [36, Section 5] for its detailed construction and the proofs of the properties.

We start by defining

$$
\varphi^{ \pm}(t):=\varphi_{B_{2 r}}^{ \pm}(t), \quad t_{1}:=\left(\varphi^{-}\right)^{-1}(\omega(2 r)) \quad \text { and } \quad t_{2}:=\left(\varphi^{-}\right)^{-1}\left(\left|B_{2 r}\right|^{-1}\right) .
$$

Note that $t_{1} \leqslant 1 \leqslant t_{2}$ from the assumptions (4.10) and (A0). Now we can define

$$
\tau_{B_{2 r}}(t):= \begin{cases}a_{1}\left(\frac{t}{t_{1}}\right)^{p-1}, & \text { if } 0 \leqslant t<t_{1}, \\ \varphi^{\prime}\left(x_{0}, t\right), & \text { if } t_{1} \leqslant t \leqslant t_{2}, \\ a_{2}\left(\frac{t}{t_{2}}\right)^{p-1}, & \text { if } t_{2}<t<\infty,\end{cases}
$$

where $a_{1}:=\varphi^{\prime}\left(x_{0}, t_{1}\right)$ and $a_{2}:=\varphi^{\prime}\left(x_{0}, t_{2}\right)$ are chosen so that $\tau_{B_{2 r}}$ is continuous. We continue defining

$$
\varphi_{B_{2 r}}(t):=\int_{0}^{t} \tau_{B_{2 r}}(s) d s .
$$

Finally, for $\eta \in C_{0}^{\infty}(\mathbb{R})$ with $\eta \geqslant 0, \operatorname{supp} \eta \subset(0,1)$ and $\|\eta\|_{1}=1$, we define the regularized Orlicz function $\tilde{\varphi}(0):=0$ and

$$
\tilde{\varphi}(t):=\int_{0}^{\infty} \varphi_{B_{2 r}}(t \sigma) \eta_{r}(\sigma-1) d \sigma=\int_{0}^{\infty} \varphi_{B_{2 r}}(s) \eta_{r t}(s-t) d s, \text { where } \eta_{r}(t):=\frac{1}{r} \eta\left(\frac{t}{r}\right) .
$$

This function $\tilde{\varphi}$ has the following properties.

Lemma 4.16 (Lemma 5.10 [36]). Let $\tilde{\varphi}$ be the regularized Orlicz function. Then

(1) $\varphi_{B_{2 r}}(t) \leqslant \tilde{\varphi}(t) \leqslant(1+c r) \varphi_{B_{2 r}}(t)$ for all $t>0$ with $c>0$ depending only on $q$, and $0 \leqslant \tilde{\varphi}(t)-\varphi\left(x_{0}, t\right) \leqslant c r \varphi^{-}(t)+c \omega(2 r) \leqslant c \varphi^{-}(t)$ for all $t \in\left[t_{1}, t_{2}\right] ;$

(2) $\tilde{\varphi} \in C^{1}([0, \infty))$ and it satisfies $(\mathrm{Inc})_{p}$, (Dec) $)_{q}$ and (A0), and $\tilde{\varphi}^{\prime}$ satisfies $(\mathrm{Inc})_{p-1}$ and $(\mathrm{Dec})_{q-1}$ and $(\mathrm{A} 0)$. In particular $\tilde{\varphi}^{\prime}(t) \approx t \tilde{\varphi}^{\prime \prime}(t)$ for all $t>0$;

(3) $\tilde{\varphi}(t) \leqslant c \varphi(x, t)$ for all $(x, t) \in B_{2 r} \times[1, \infty)$, and so $\tilde{\varphi}(t) \lesssim \varphi(x, t)+1$ for all $(x, t) \in B_{2 r} \times[0, \infty)$.

Here the constant $c$ and the implicit constants depend only on $n, p, q$ and $L$.

Now let us consider the following comparison principle for $\tilde{\varphi}$.

Lemma 4.17. Assume that $w \in W^{1, \varphi}(\Omega)$ satisfies

$$
\left\{\begin{aligned}
-\operatorname{div}\left(\frac{\tilde{\varphi}^{\prime}(|\nabla \psi|)}{|\nabla \psi|} \nabla \psi\right) & \leqslant-\operatorname{div}\left(\frac{\tilde{\varphi}^{\prime}(|\nabla w|)}{|\nabla w|} \nabla w\right) & \text { in } \quad B_{r}, \\
\psi & \leqslant w & \text { on } \partial B_{r},
\end{aligned}\right.
$$

in the weak sense, that is, $(\psi-w)_{+} \in W^{1, \tilde{\varphi}}\left(B_{r}\right)$ and

$$
\int_{B_{r}}\left(\frac{\tilde{\varphi}^{\prime}(|\nabla \psi|)}{|\nabla \psi|} \nabla \psi-\frac{\tilde{\varphi}^{\prime}(|\nabla w|)}{|\nabla w|} \nabla w\right) \cdot \nabla \eta d x \leqslant 0 \text { for all } \eta \in W^{1, \tilde{\varphi}}\left(B_{r}\right) \text { with } \eta \geqslant 0 \text {. }
$$

Then we have $\psi \leqslant w$ a.e. in $B_{r}$.

Proof. By taking $\eta=(\psi-w)_{+}$as a test function to the above weak formulation, we see

$$
\int_{B_{r} \cap\{\psi>w\}}\left(\frac{\tilde{\varphi}^{\prime}(|\nabla \psi|)}{|\nabla \psi|} \nabla \psi-\frac{\tilde{\varphi}^{\prime}(|\nabla w|)}{|\nabla w|} \nabla w\right) \cdot(\nabla \psi-\nabla w) d x \leqslant 0 .
$$


Then using first (4) and then (2) of Lemma 2.10, we obtain

$$
\begin{aligned}
& \int_{B_{r} \cap\{\psi>w\}} \tilde{\varphi}(|\nabla \psi-\nabla w|) d x \\
& \lesssim \kappa \int_{B_{r} \cap\{\psi>w\}} \tilde{\varphi}(|\nabla \psi|)+\tilde{\varphi}(|\nabla w|) d x \\
& \quad+\kappa^{-1} \int_{B_{r} \cap\{\psi>w\}} \frac{\tilde{\varphi}^{\prime}(|\nabla \psi|+|\nabla w|)}{|\nabla \psi|+|\nabla w|}|\nabla \psi-\nabla w|^{2} d x \\
& \lesssim \kappa \int_{B_{r} \cap\{\psi>w\}} \tilde{\varphi}(|\nabla \psi|)+\tilde{\varphi}(|\nabla w|) d x \\
& \quad+\kappa^{-1} \int_{B_{r} \cap\{\psi>w\}}\left(\frac{\tilde{\varphi}^{\prime}(|\nabla \psi|)}{|\nabla \psi|} \nabla \psi-\frac{\tilde{\varphi}^{\prime}(\nabla w \mid)}{|\nabla w|} \nabla w\right) \cdot(\nabla \psi-\nabla w) d x \\
& \leqslant \kappa \int_{B_{r} \cap\{\psi>w\}} \tilde{\varphi}(|\nabla \psi|)+\tilde{\varphi}(|\nabla w|) d x
\end{aligned}
$$

for any $\kappa \in(0,1)$. Since $\kappa$ is arbitrary, we have that $\psi \leqslant w$ a.e. in $B_{r}$.

Next we define two equations and corresponding solutions $w$ and $v$ to which we compare our solution $u$ to the obstacle problem (2.4). We will prove some energy estimates of $w$ and $v$ with respect to $u$ and regularized Orlicz function $\tilde{\varphi}$ in Lemma 4.20.

Let $u \in \mathcal{K}_{\psi}^{\varphi}(\Omega)$ be a solution to the $\mathcal{K}_{\psi}^{\varphi}(\Omega)$-obstacle problem and $B_{2 r} \subset \Omega$ with $r>0$ satisfying (4.10). Let us consider the unique weak solution $w \in W^{1, \tilde{\varphi}}\left(B_{r}\right)$ of

$$
\left\{\begin{array}{rlrl}
-\operatorname{div}\left(\frac{\tilde{\varphi}^{\prime}(|\nabla w|)}{|\nabla w|} \nabla w\right) & =-\operatorname{div}\left(\frac{\tilde{\varphi}^{\prime}(|\nabla \psi|)}{|\nabla \psi|} \nabla \psi\right) & \text { in } \quad B_{r} \\
w & =u & \text { on } & \partial B_{r}
\end{array}\right.
$$

and the unique weak solution $v \in W^{1, \tilde{\varphi}}\left(B_{r}\right)$ of

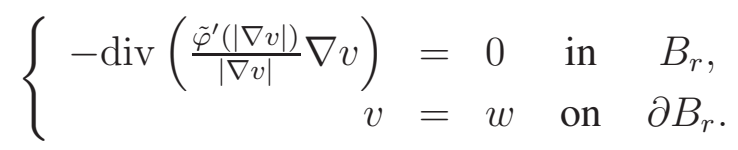

From now on, we set $\overline{\nabla \psi}:=\sup _{\Omega}|\nabla \psi|$ for simplicity.

Lemma 4.20. For $w$ and $v$ defined in (4.18) and (4.19) we have the energy estimates:

$$
f_{B_{r}} \tilde{\varphi}(|\nabla w|) d x \leqslant c\left(f_{B_{r}} \tilde{\varphi}(|\nabla u|) d x+f_{B_{r}} \tilde{\varphi}(|\nabla \psi|) d x\right) \leqslant c\left(f_{B_{r}} \tilde{\varphi}(|\nabla u|) d x+1\right)
$$

and

$$
f_{B_{r}} \tilde{\varphi}(|\nabla v|) d x \leqslant c f_{B_{r}} \tilde{\varphi}(|\nabla w|) d x \leqslant c\left(f_{B_{r}} \tilde{\varphi}(|\nabla u|) d x+1\right)
$$

where $c=c(n, p, q, L, \overline{\nabla \psi})>0$.

Proof. Testing with $w-u \in W_{0}^{1, \tilde{\varphi}}\left(B_{r}\right)$ to the weak formulation, the equation (4.18) yields

$$
f_{B_{r}} \frac{\tilde{\varphi}^{\prime}(|\nabla w|)}{|\nabla w|} \nabla w \cdot \nabla w d x=f_{B_{r}} \frac{\tilde{\varphi}^{\prime}(|\nabla w|)}{|\nabla w|} \nabla w \cdot \nabla u+\frac{\tilde{\varphi}^{\prime}(|\nabla \psi|)}{|\nabla \psi|} \nabla \psi \cdot \nabla(w-u) d x .
$$


Applying $\tilde{\varphi}^{\prime}(t) t \approx \tilde{\varphi}(t)$ in Lemma 2.10 (1), Lemma 2.11 (4)-(5), and (aDec) $)_{q}$, we obtain

$$
\begin{aligned}
f_{B_{r}} \frac{\tilde{\varphi}^{\prime}(|\nabla w|)}{|\nabla w|} \nabla w \cdot \nabla w d x & \leqslant c f_{B_{r}} \frac{\tilde{\varphi}(|\nabla w|)}{|\nabla w|}|\nabla u|+\frac{\tilde{\varphi}(|\nabla \psi|)}{|\nabla \psi|}(|\nabla w|+|\nabla u|) d x \\
& \leqslant \frac{1}{2} f_{B_{r}} \tilde{\varphi}(|\nabla w|) d x+c f_{B_{r}} \tilde{\varphi}(|\nabla u|)+\tilde{\varphi}(|\nabla \psi|) d x .
\end{aligned}
$$

Recalling Lemma 2.10 (1) again, we can move the first term on the right-hand side to the left-hand side. Finally, estimating $|\nabla \psi|$ by $\overline{\nabla \psi}$ we have proven (4.21).

The second energy estimate (4.22) follows immediately since $v$ as a solution minimizes the corresponding energy integral and $v$ and $w$ have the same boundary values in the Sobolev sense.

As $v$ is the solution of the $\varphi$-Laplacian equation, it is known to have $C^{1, \alpha_{0}}$-regularity for some $\alpha_{0}>0$ from [39] (see also [36, Lemma 4.12]). Additionally, for any $B\left(x_{0}, \varrho\right) \subset B_{r}$, we have

$$
\sup _{B\left(x_{0}, \varrho / 2\right)}|\nabla v| \leqslant c f_{B\left(x_{0}, \varrho\right)}|\nabla v| d x
$$

and for any $\tau \in(0,1)$

$$
f_{B\left(x_{0}, \tau \varrho\right)}\left|\nabla v-(\nabla v)_{B\left(x_{0}, \tau \varrho\right)}\right| d x \leqslant c \tau^{\alpha_{0}} f_{B\left(x_{0}, \varrho\right)}|\nabla v| d x
$$

where $\alpha_{0} \in(0,1)$ and $c=c(n, p, q)>0$.

4.3. Calderón-Zygmund type estimates. We also need the following reverse type estimate and Calderón-Zygmund type estimate for the equation (4.18). Here we rely on similar results in [36, Lemma 4.15]. As the proofs of these results are almost identical to the original one, we give just sketches of the proofs. The second lemma is an application suitable for the obstacle problem of the more abstract Calderón-Zygmund type estimates outlined in the first lemma.

The following is Calderón-Zygmund type estimates with a ball $B_{r}$ with radius $r$.

Lemma 4.25. Let $\varphi \in \Phi_{c} \cap C^{1}([0, \infty)) \cap C^{2}((0, \infty))$ with $\varphi^{\prime}$ satisfying $(\mathrm{Inc})_{p-1}$ and $(\mathrm{Dec})_{q-1}$ for some $1<p \leqslant q$, and $\left|B_{r}\right| \leqslant 1$. If $w$ is a solution to (4.18), then there exists a constant $c=c\left(n, p, q, p_{1}, q_{1}, L\right)>0$ such that

$$
\|\varphi(|\nabla w|)\|_{L^{\theta}\left(B_{r}\right)} \leqslant c\left(\|\varphi(|\nabla \psi|)\|_{L^{\theta}\left(B_{r}\right)}+\|\varphi(|\nabla u|)\|_{L^{\theta}\left(B_{r}\right)}\right)
$$

for any $\theta \in \Phi_{w}\left(B_{r}\right)$ satisfying (A0), (A1), (aInc) $)_{p_{1}}$ and $(\mathrm{aDec})_{q_{1}}$ with constant $L \geqslant 1$ and $1<p_{1} \leqslant q_{1}$.

Moreover, fix $\kappa>0$ and assume that $\int_{B_{r}} \theta(x, \varphi(|\nabla u|))+\theta(x, \varphi(|\nabla \psi|)) d x \leqslant \kappa$. Then

$$
f_{B_{r}} \theta(x \cdot \varphi(|\nabla w|)) d x \leqslant c\left(\kappa^{\frac{q_{1}}{p_{1}}-1}+1\right)\left(f_{B_{r}} \theta(x, \varphi(|\nabla u|))+\theta(x, \varphi(|\nabla \psi|)) d x+1\right)
$$

for some $c \geqslant 0$ depending on $n, p, q, p_{1}, q_{1}$ and $L$.

Proof. In the same way to [36, Theorem B.1 in Appendix B] (also see [42]), we obtain that for any $s \in(0, \infty)$ and Muckenhoupt weight $\mu \in A_{s}$,

$$
\int_{\Omega} \varphi(|\nabla w|)^{s} \mu(x) d x \leqslant c\left(\int_{\Omega} \varphi(|\nabla \psi|)^{s} \mu(x) d x+\int_{\Omega} \varphi(|\nabla u|)^{s} \mu(x) d x\right)
$$


for some constant $c=c\left(n, p, q, s,[\mu]_{A_{s}}\right)>0$ but we need to replace (B.2) by

$$
\operatorname{div}\left(\frac{\varphi^{\prime}(|\nabla w|)}{|\nabla w|} \nabla w\right)=\operatorname{div}\left(\frac{\varphi^{\prime}(|\nabla \psi|)}{|\nabla \psi|} \nabla \psi\right) \text { in } \Omega \text { with } w=u \text { on } \partial \Omega,
$$

where $\Omega \subset \mathbb{R}^{n}$ is a Reifenberg flat domain. In addition, to compare this equation with an equation having zero boundary values on $\partial \Omega$ in a local region near boundary, we add the one more assumption

$$
f_{\Omega_{5}} \varphi(|\nabla \psi|) d x \leqslant \delta
$$

to (B.7) and continue similarly with the proofs in Lemmas B.5 and B.11 to derive (4.28). Hence (4.28) with $\Omega=B_{r}$ implies the desired norm inequality (4.26) via the extrapolation result for the generalized Orlicz function in [30, Corollary 5.3.4].

The modular inequality (4.27) follows by modifying the proof of [36, Lemma 4.15] slightly, that is replacing the definition of constant $M$ with

$$
M:=\left(\theta^{-}\right)^{-1}\left(\int_{B_{r}} \theta(x, \varphi(|\nabla u|))+\theta(x, \varphi(|\nabla \psi|)) d x\right) .
$$

Then in similar fashion we obtain

$$
\int_{B_{r}} \bar{\theta}(x, \bar{\varphi}(\mid \nabla u))+\bar{\theta}(x, \bar{\varphi}(\mid \nabla \psi)) d x \leqslant 1 \Rightarrow\|\bar{\varphi}(|\nabla u|)\|_{L^{\bar{\theta}\left(B_{r}\right)}}+\|\bar{\varphi}(|\nabla \psi|)\|_{L^{\bar{\theta}}\left(B_{r}\right)} \leqslant 2
$$

and the rest follows line by line.

Lemma 4.29. We have that

$$
\left(f_{B_{r}} \varphi(x,|\nabla u|)^{1+\sigma_{0}} d x\right)^{\frac{1}{1+\sigma_{0}}} \lesssim \tilde{\varphi}\left(f_{B_{2 r}}|\nabla u| d x\right)+1
$$

and

$$
\begin{aligned}
f_{B_{r}} \varphi(x,|\nabla w|) d x & \leqslant\left(f_{B_{r}} \varphi(x,|\nabla w|)^{1+\frac{\sigma_{0}}{2}} d x\right)^{\frac{2}{2+\sigma_{0}}} \\
& \lesssim\left(f_{B_{r}} \varphi(x,|\nabla u|)^{1+\frac{\sigma_{0}}{2}} d x+1\right)^{\frac{2}{2+\sigma_{0}}}
\end{aligned}
$$

where the implicit constant depends on $n, p, q, L$ and $\overline{\nabla \psi}$.

Proof. Since (wVA1) implies (A1), we have from (4.8) in Lemma 4.6 that

$$
\begin{aligned}
& \left(f_{B_{r}} \varphi(x,|\nabla u|)^{1+\sigma_{0}} d x\right)^{1 /\left(1+\sigma_{0}\right)} \\
& \quad \lesssim \varphi_{B_{2 r}}^{-}\left(f_{B_{2 r}}|\nabla u| d x\right)+\left(f_{B_{2 r}} \varphi(x,|\nabla \psi|)^{1+\sigma_{0}} d x\right)^{1 /\left(1+\sigma_{0}\right)}+1
\end{aligned}
$$

Using $\overline{\nabla \psi}$ we can absorb the second term on the right-hand side to the implicit constant. When $f_{B_{2 r}}|\nabla u| d x \leqslant 1$, we obtain (4.30) by finiteness of $\varphi_{B_{2 r}}^{-}$. In the other case that $f_{B_{2 r}}|\nabla u| d x>1$, we have

$$
1<f_{B_{2 r}}|\nabla u| d x \leqslant\left(\varphi_{B_{2 r}}^{-}\right)^{-1}\left(f_{B_{2 r}} \varphi_{B_{2 r}}^{-}(|\nabla u|) d x\right) \leqslant\left(\varphi_{B_{2 r}}^{-}\right)^{-1}\left(\left|B_{2 r}\right|^{-1}\right)=t_{2},
$$


where $t_{2}$ is defined in (4.13). Thus, Lemma 4.16 (1) yields that

$$
\begin{aligned}
\left(f_{B_{r}} \varphi(x,|\nabla u|)^{1+\sigma_{0}} d x\right)^{1 /\left(1+\sigma_{0}\right)} & \lesssim \varphi_{B_{2 r}}^{-}\left(f_{B_{2 r}}|\nabla u| d x\right)+1 \\
& \lesssim \varphi\left(x_{0}, f_{B_{2 r}}|\nabla u| d x\right)+1 \lesssim \tilde{\varphi}\left(f_{B_{2 r}}|\nabla u| d x\right)+1
\end{aligned}
$$

which is (4.30).

In order to prove (4.31), let us consider the function $\theta \in \Phi_{w}\left(B_{r}\right)$ which is given in

$$
\theta(x, t):=\left[\varphi\left(x, \tilde{\varphi}^{-1}(t)\right)\right]^{1+\frac{\sigma_{0}}{2}}
$$

for any fixed $\sigma_{0} \in(0,1)$. Applying the Calderón-Zygmund estimates (4.27) and local boundedness of $|\nabla \psi|$ we have that

$$
\begin{aligned}
f_{B_{r}} \varphi(x,|\nabla w|)^{1+\frac{\sigma_{0}}{2}} d x & =f_{B_{r}} \theta(x, \tilde{\varphi}(|\nabla w|)) d x \\
& \lesssim f_{B_{r}} \theta(x, \tilde{\varphi}(|\nabla u|)) d x+f_{B_{r}} \theta(x, \tilde{\varphi}(|\nabla \psi|)) d x+1 \\
& =f_{B_{r}} \varphi(x,|\nabla u|)^{1+\frac{\sigma_{0}}{2}} d x+f_{B_{r}} \varphi(x,|\nabla \psi|)^{1+\frac{\sigma_{0}}{2}} d x+1 \\
& \lesssim f_{B_{r}} \varphi(x,|\nabla u|)^{1+\frac{\sigma_{0}}{2}} d x+1
\end{aligned}
$$

The desired inequality (4.31) follows by taking the $\frac{2}{2+\sigma_{0}}$ 'th root from both sides and applying Hölder's inequality on the left hand side.

4.4. Comparison estimates. Now we are ready to prove comparison estimates between different solutions. These are the main ingredients for the proof of Theorem 2.7. Much of the proof is similar to the case without any obstacle and these omitted details can be found in [36].

Lemma 4.32. Suppose that $w$ is a solution to the equation (4.18), $u$ is a solution to the equation (2.4) and $B_{2 r} \Subset \Omega$. Then

$$
f_{B_{r}}|\nabla u-\nabla w| d x \leqslant c\left(\omega(2 r)^{p / q}+r^{\gamma}+r^{\beta}\right)^{\frac{1}{2 q}}\left(f_{B_{2 r}}|\nabla u| d x+1\right)
$$

for some $\gamma=(n, p, q, L) \in(0,1)$ and some constant $c=c\left(n, p, q, L,[\nabla \psi]_{\beta}, \overline{\nabla \psi}\right)>0$, where $\omega$ is from the assumption (wVA1).

Proof. From (4.18), we see that

$$
\int_{B_{r}} \frac{\tilde{\varphi}^{\prime}(|\nabla w|)}{|\nabla w|} \nabla w \cdot(\nabla w-\nabla u) d x=\int_{B_{r}} \frac{\tilde{\varphi}^{\prime}(|\nabla \psi|)}{|\nabla \psi|} \nabla \psi \cdot(\nabla w-\nabla u) d x,
$$

by taking $w-u \in W_{0}^{1, \tilde{\varphi}}\left(B_{r}\right)$ as a test function to the weak formulation.

Recalling Lemma 4.17 and setting $w=u$ in $\Omega \backslash B_{r}$, we have that $w \in W^{1, \varphi}(\Omega)$ and $w \geqslant \psi$ a.e. in $\Omega$, and so $w \in \mathcal{K}_{\psi}^{\varphi}(\Omega)$. Therefore by taking $\eta:=w-u$ as a test function in (2.4),

$$
\int_{B_{r}} \frac{\partial_{t} \varphi(x,|\nabla u|)}{|\nabla u|} \nabla u \cdot(\nabla w-\nabla u) d x \geqslant 0
$$


From Lemma 2.10 (3) and (4.33), we then derive that

$$
\begin{aligned}
& \int_{B_{r}} \tilde{\varphi}^{\prime \prime}(|\nabla u|+|\nabla w|)|\nabla u-\nabla w|^{2} d x \\
& \lesssim \int_{B_{r}} \tilde{\varphi}(|\nabla u|)-\tilde{\varphi}(|\nabla w|)-\frac{\tilde{\varphi}^{\prime}(|\nabla w|)}{|\nabla w|} \nabla w \cdot(\nabla u-\nabla w) d x \\
& =\int_{B_{r}} \tilde{\varphi}(|\nabla u|)-\tilde{\varphi}(|\nabla w|) d x+\int_{B_{r}} \frac{\tilde{\varphi}^{\prime}(|\nabla \psi|)}{|\nabla \psi|} \nabla \psi \cdot(\nabla w-\nabla u) d x \\
& =\int_{B_{r}} \tilde{\varphi}(|\nabla u|)-\varphi(x,|\nabla u|) d x+\int_{B_{r}} \varphi(x,|\nabla u|)-\varphi(x,|\nabla w|) d x \\
& \quad+\int_{B_{r}} \varphi(x,|\nabla w|)-\tilde{\varphi}(|\nabla w|) d x+\int_{B_{r}} \frac{\tilde{\varphi}^{\prime}(|\nabla \psi|)}{|\nabla \psi|} \nabla \psi \cdot(\nabla w-\nabla u) d x .
\end{aligned}
$$

Here, applying Lemma 2.10 (3) pointwise again with (4.34), we see that

$$
\begin{aligned}
& \int_{B_{r}} \varphi(x,|\nabla u|)-\varphi(x,|\nabla w|) d x \\
& \lesssim \int_{B_{r}} \frac{\partial_{t} \varphi(x,|\nabla u|)}{|\nabla u|} \nabla u \cdot(\nabla u-\nabla w) d x-\int_{B_{r}} \frac{\partial_{t} \varphi(x,|\nabla u|+|\nabla w|)}{|\nabla u|+|\nabla w|}|\nabla u-\nabla w|^{2} d x \\
& \leqslant-\int_{B_{r}} \frac{\partial_{t} \varphi(x,|\nabla u|+|\nabla w|)}{|\nabla u|+|\nabla w|}|\nabla u-\nabla w|^{2} d x \leqslant 0
\end{aligned}
$$

since $\varphi$ is increasing by assumption. Combining the above two inequalities, we obtain that

$$
\begin{aligned}
& f_{B_{r}} \tilde{\varphi}^{\prime \prime}(|\nabla u|+|\nabla w|)|\nabla u-\nabla w|^{2} d x \\
& \leqslant \underbrace{f_{B_{r}} \tilde{\varphi}(|\nabla u|)-\varphi(x,|\nabla u|) d x}_{=: I_{1}}+\underbrace{f_{B_{r}} \varphi(x,|\nabla w|)-\tilde{\varphi}(|\nabla w|) d x}_{=: I_{2}} \\
& +\underbrace{f_{B_{r}} \frac{\tilde{\varphi}^{\prime}(|\nabla \psi|)}{|\nabla \psi|} \nabla \psi \cdot(\nabla w-\nabla u) d x}_{:=I_{3}} .
\end{aligned}
$$

On the other hand, using Lemma 2.10 (4) and (4.21), we infer that

$$
\begin{aligned}
& f_{B_{r}} \tilde{\varphi}(|\nabla u-\nabla w|) d x \\
& \lesssim \kappa f_{B_{r}} \tilde{\varphi}(|\nabla u|)+\tilde{\varphi}(|\nabla w|) d x+\kappa^{-1} f_{B_{r}} \tilde{\varphi}^{\prime \prime}(|\nabla u|+|\nabla w|)|\nabla u-\nabla w|^{2} d x \\
& \lesssim \kappa\left(f_{B_{r}} \tilde{\varphi}(|\nabla u|) d x+1\right)+\kappa^{-1}\left(I_{1}+I_{2}+I_{3}\right)
\end{aligned}
$$

for any $\kappa \in(0, \infty)$.

To estimate $I_{2}$, we adopt the method in the proof of [36, Lemma 6.2]. We start by splitting $B_{r}$ into three parts:

$$
\begin{aligned}
& E_{1}:=B_{r} \cap\left\{\varphi^{-}(|\nabla w|) \leqslant \omega(2 r)\right\} \\
& E_{2}:=B_{r} \cap\left\{\omega(2 r)<\varphi^{-}(|\nabla w|) \leqslant\left|B_{2 r}\right|^{-1+\varepsilon_{0}}\right\} \\
& E_{3}:=B_{r} \cap\left\{\left|B_{2 r}\right|^{-1+\varepsilon_{0}}<\varphi^{-}(|\nabla w|)\right\} .
\end{aligned}
$$


In the set $E_{1}$ assumptions $(\mathrm{Dec})_{q}$ and (A0) of $\varphi$ yield that $|\nabla w| \lesssim \omega(2 r)^{\frac{1}{q}}$. Now (Inc) $)_{p}$ and (A0) of $\varphi$ and $\tilde{\varphi}$ imply

$$
f_{B_{r}}|\varphi(x,|\nabla w|)-\tilde{\varphi}(|\nabla w|)| \chi_{E_{1}} d x \lesssim \omega(2 r)^{p / q} f_{B_{r}} \chi_{E_{1}} d x \leqslant \omega(2 r)^{p / q} .
$$

In the set $E_{2}$ we get

$$
\omega(2 r)<\varphi^{-}(|\nabla w|) \leqslant\left|B_{2 r}\right|^{-1+\varepsilon_{0}}<\left|B_{2 r}\right|^{-1}
$$

which implies that $t_{1}<|\nabla w|<t_{2}$. Therefore we can use (wVA1) and Lemma 4.16 (1) to get

$$
\begin{aligned}
|\varphi(x,|\nabla w|)-\tilde{\varphi}(|\nabla w|)| & \leqslant\left|\varphi(x,|\nabla w|)-\varphi\left(x_{0},|\nabla w|\right)\right|+\left|\varphi\left(x_{0},|\nabla w|\right)-\tilde{\varphi}(|\nabla w|)\right| \\
& \leqslant \varphi^{+}(|\nabla w|)-\varphi^{-}(|\nabla w|)+\tilde{\varphi}(|\nabla w|)-\varphi\left(x_{0},|\nabla w|\right) \\
& \lesssim(1+\omega(2 r)) \varphi^{-}(|\nabla w|)+\omega(2 r)-\varphi^{-}(|\nabla w|)+r \varphi^{-}(|\nabla w|)+\omega(2 r) \\
& \lesssim(r+\omega(2 r)) \varphi^{-}(|\nabla w|)+\omega(2 r) .
\end{aligned}
$$

Note that this is the only place, where the assumption (wVA1) is needed. Now using (4.31) and (4.30) we see that

$$
f_{B_{r}}|\varphi(x,|\nabla w|)-\tilde{\varphi}(\mid \nabla w)| \chi_{E_{2}} d x \lesssim(r+\omega(2 r))\left(\tilde{\varphi}\left(f_{B_{2 r}}|\nabla u| d x\right)+1\right) .
$$

For $E_{3}$ we have the inequality $1<\left|B_{2 r}\right|^{1-\varepsilon_{0}} \varphi^{-}(|\nabla w|)$, which implies with Lemma 4.16 (3)

$$
\begin{aligned}
|\varphi(x,|\nabla w|)-\tilde{\varphi}(|\nabla w|)| & \lesssim \varphi(x,|\nabla w|)+1 \lesssim \varphi(x,|\nabla w|) \\
& \leqslant\left[\left|B_{2 r}\right|^{1-\varepsilon_{0}} \varphi^{-}(|\nabla w|)\right]^{\sigma_{0} / 2} \varphi(x,|\nabla w|) \\
& \lesssim r^{n\left(1-\varepsilon_{0}\right) \sigma_{0} / 2} \varphi(x,|\nabla w|)^{1+\frac{\sigma_{0}}{2}}
\end{aligned}
$$

Letting

$$
\varepsilon_{0}:=\frac{\sigma_{0}}{2\left(2+\sigma_{0}\right)}
$$

and integrating both sides over the set $E_{3}$ we obtain

$$
f_{B_{r}}|\varphi(x,|\nabla w|)-\tilde{\varphi}(|\nabla w|)| \chi_{E_{3}} d x \lesssim r^{\frac{n\left(4+\sigma_{0}\right) \sigma_{0}}{4\left(2+\sigma_{0}\right)}}\left(f_{B_{r}} \varphi(x,|\nabla w|)^{1+\frac{\sigma_{0}}{2}} d x\right)^{\frac{2}{2+\sigma_{0}}+\frac{\sigma_{0}}{2+\sigma_{0}}} .
$$

Firstly, (4.31) and (4.30) imply that

$$
\left(f_{B_{r}} \varphi(x,|\nabla w|)^{1+\frac{\sigma_{0}}{2}} d x\right)^{\frac{2}{2+\sigma_{0}}} \lesssim \tilde{\varphi}\left(f_{B_{2 r}}|\nabla u| d x\right)+1
$$

Secondly, by (4.12)

$$
\left(f_{B_{r}} \varphi(x,|\nabla w|)^{1+\frac{\sigma_{0}}{2}} d x\right)^{\frac{\sigma_{0}}{2+\sigma_{0}}} \leqslant\left|B_{r}\right|^{-\frac{\sigma_{0}}{2+\sigma_{0}}} \lesssim r^{-\frac{n \sigma_{0}}{2+\sigma_{0}}} .
$$

Using three previous estimates we find that

$$
f_{B_{r}}|\varphi(x,|\nabla w|)-\tilde{\varphi}(|\nabla w|)| \chi_{E_{3}} d x \lesssim r^{\frac{n \sigma_{0}^{2}}{4\left(2+\sigma_{0}\right)}}\left(\tilde{\varphi}\left(f_{B_{2 r}}|\nabla u| d x\right)+1\right) .
$$


Combining (4.35), (4.36) and (4.37) together, we have arrived at

$$
\begin{aligned}
\left|I_{2}\right| & \leqslant f_{B_{r}}|\varphi(x,|\nabla w|)-\tilde{\varphi}(|\nabla w|)| d x \\
& \lesssim\left(\omega(2 r)^{p / q}+r+r^{\frac{n \sigma_{0}^{2}}{4\left(2+\sigma_{0}\right)}}\right)\left(\tilde{\varphi}\left(f_{B_{2 r}}|\nabla u| d x\right)+1\right) \\
& \lesssim\left(\omega(2 r)^{p / q}+r^{\gamma}\right)\left(\tilde{\varphi}\left(f_{B_{2 r}}|\nabla u| d x\right)+1\right)
\end{aligned}
$$

where $\gamma=\min \left\{1, \frac{n \sigma_{0}^{2}}{4\left(2+\sigma_{0}\right)}\right\}$. The estimate for $I_{1}$ is analogous to $I_{2}$ with $\nabla u$ instead of $\nabla w$.

We continue with estimating $I_{3}$. We start by calculating

$$
\begin{aligned}
\partial \tilde{\varphi}(|x|)-\partial \tilde{\varphi}(y) & =\int_{0}^{1} \frac{d}{d t} \partial \tilde{\varphi}(t x+(1-t) y) d t \leqslant|x-y| \int_{0}^{1} \tilde{\varphi}^{\prime \prime}(|x|+|y|) d t \\
& \approx|x-y| \frac{\tilde{\varphi}^{\prime}(|x|+|y|)}{|x|+|y|} .
\end{aligned}
$$

Identifying $x=\nabla \psi$ and $y=\nabla \psi\left(x_{0}\right)$, where $x_{0}$ is the center of $B_{r}$, and using Lemma 2.10 (2) we have

$$
\begin{aligned}
\left|I_{3}\right| & =\left|f_{B_{r}} \frac{\tilde{\varphi}^{\prime}(|\nabla \psi|)}{|\nabla \psi|} \nabla \psi \cdot(\nabla w-\nabla u) d x\right| \\
& =\left|f_{B_{r}}\left[\frac{\tilde{\varphi}^{\prime}(|\nabla \psi|)}{|\nabla \psi|} \nabla \psi-\frac{\tilde{\varphi}^{\prime}\left(\left|\nabla \psi\left(x_{0}\right)\right|\right)}{\left|\nabla \psi\left(x_{0}\right)\right|} \nabla \psi\left(x_{0}\right)\right] \cdot(\nabla w-\nabla u) d x\right| \\
& \lesssim f_{B_{r}} \frac{\tilde{\varphi}^{\prime}\left(|\nabla \psi|+\left|\nabla \psi\left(x_{0}\right)\right|\right)}{|\nabla \psi|+\left|\nabla \psi\left(x_{0}\right)\right|}\left|\nabla \psi-\nabla \psi\left(x_{0}\right)\right||\nabla w-\nabla u| d x .
\end{aligned}
$$

Since $\tilde{\varphi}^{\prime}$ satisfies (Inc) $)_{p-1}$, we note that if $p \geqslant 2$, then $\tilde{\varphi}^{\prime}(t) / t$ is non-decreasing for $t \in$ $(0, \infty)$. Then

$$
\begin{aligned}
\left|I_{3}\right| & \lesssim f_{B_{r}} \frac{\tilde{\varphi}^{\prime}\left(|\nabla \psi|+\left|\nabla \psi\left(x_{0}\right)\right|\right)}{|\nabla \psi|+\left|\nabla \psi\left(x_{0}\right)\right|}\left|\nabla \psi-\nabla \psi\left(x_{0}\right)\right||\nabla w-\nabla u| d x \\
& \lesssim r^{\beta} f_{B_{r}}|\nabla u|+|\nabla w| d x \lesssim r^{\beta}\left(f_{B_{r}} \tilde{\varphi}(|\nabla u|) d x+1\right) .
\end{aligned}
$$

If $p<2$, we deduce that

$$
\begin{aligned}
\left|I_{3}\right| & \lesssim f_{B_{r}} \frac{\tilde{\varphi}^{\prime}\left(|\nabla \psi|+\left|\nabla \psi\left(x_{0}\right)\right|\right)}{|\nabla \psi|+\left|\nabla \psi\left(x_{0}\right)\right|}\left|\nabla \psi-\nabla \psi\left(x_{0}\right)\right||\nabla w-\nabla u| d x \\
& \lesssim f_{B_{r}} \frac{\tilde{\varphi}^{\prime}\left(|\nabla \psi|+\left|\nabla \psi\left(x_{0}\right)\right|\right)}{|\nabla \psi|+\left|\nabla \psi\left(x_{0}\right)\right|}\left(|\nabla \psi|+\left|\nabla \psi\left(x_{0}\right)\right|\right)^{2-p}\left|\nabla \psi-\nabla \psi\left(x_{0}\right)\right|^{p-1}|\nabla w-\nabla u| d x \\
& =f_{B_{r}} \frac{\tilde{\varphi}^{\prime}\left(|\nabla \psi|+\left|\nabla \psi\left(x_{0}\right)\right|\right)}{\left(|\nabla \psi|+\left|\nabla \psi\left(x_{0}\right)\right|\right)^{p-1}}\left|\nabla \psi-\nabla \psi\left(x_{0}\right)\right|^{p-1}|\nabla w-\nabla u| d x \\
& \lesssim r^{\beta(p-1)} f_{B_{r}}|\nabla u|+|\nabla w| d x \lesssim r^{\beta(p-1)}\left(f_{B_{r}} \tilde{\varphi}(|\nabla u|) d x+1\right),
\end{aligned}
$$

since $\tilde{\varphi}^{\prime}(t) / t^{p-1}$ is non-decreasing for $t \in(0, \infty)$. In turn, we conclude that

$$
\left|I_{3}\right| \lesssim r^{\beta}\left(f_{B_{r}} \tilde{\varphi}(|\nabla u|) d x+1\right)
$$

where the implicit constant depends on $n, p, q, L,[\nabla \psi]_{\beta}, \overline{\nabla \psi}$. 
Note from (4.30) and Lemma 4.16 (3) that

$$
\begin{aligned}
f_{B_{r}} \tilde{\varphi}(|\nabla u|) d x & \lesssim f_{B_{r}} \varphi(x,|\nabla u|) d x+1 \\
& \leqslant\left(f_{B_{r}} \varphi(x,|\nabla u|)^{1+\sigma_{0}} d x\right)^{\frac{1}{1+\sigma_{0}}}+1 \lesssim \tilde{\varphi}\left(f_{B_{2 r}}|\nabla u| d x\right)+1 .
\end{aligned}
$$

Hence, for any $\kappa \in(0,1)$, we conclude that

$$
\begin{aligned}
& f_{B_{r}} \tilde{\varphi}(|\nabla u-\nabla w|) d x \\
& \lesssim \kappa\left(f_{B_{r}} \tilde{\varphi}(|\nabla u|) d x+1\right)+\kappa^{-1}\left(\omega(2 r)+r^{\gamma}+r^{\beta}\right)\left[\tilde{\varphi}\left(f_{B_{2 r}}|\nabla u| d x\right)+1\right] \\
& \lesssim\left(\kappa+\kappa^{-1}\left[\omega(2 r)^{p / q}+r^{\gamma}+r^{\beta}\right]\right)\left[\tilde{\varphi}\left(f_{B_{2 r}}|\nabla u| d x\right)+1\right] .
\end{aligned}
$$

By taking $\kappa:=\left(\omega(2 r)^{p / q}+r^{\gamma}+r^{\beta}\right)^{\frac{1}{2}}$, we then conclude that

$$
f_{B_{r}} \tilde{\varphi}(|\nabla u-\nabla w|) d x \lesssim\left(\omega(2 r)^{p / q}+r^{\gamma}+r^{\beta}\right)^{\frac{1}{2}}\left[\tilde{\varphi}\left(f_{B_{2 r}}|\nabla u| d x\right)+1\right] .
$$

In turn, by Jensen's inequality and $(\mathrm{aDec})_{q}$ of $\tilde{\varphi}$, we obtain that

$$
\begin{aligned}
\tilde{\varphi}\left(f_{B_{r}}|\nabla u-\nabla w| d x\right) & \lesssim f_{B_{r}} \tilde{\varphi}(|\nabla u-\nabla w|) d x \\
& \lesssim \tilde{\varphi}\left(\left(\omega(2 r)^{p / q}+r^{\gamma}+r^{\beta}\right)^{\frac{1}{2 q}}\left[f_{B_{2 r}}|\nabla u| d x+1\right]\right),
\end{aligned}
$$

which implies the claim since $\tilde{\varphi}$ is strictly increasing.

With similar arguments we get a comparison estimate between $w$ and $v$.

Lemma 4.38. Suppose that $w$ is a solution to the equation (4.18), $v$ is a solution to the equation (4.19) and $B_{2 r} \Subset \Omega$. Then

$$
f_{B_{r}}|\nabla w-\nabla v| d x \leqslant c r^{\frac{\beta}{2 q}}\left(f_{B_{2 r}}|\nabla u| d x+1\right)
$$

for some constant $c=c\left(n, p, q, L,[\nabla \psi]_{\beta}, \overline{\nabla \psi}\right)>0$.

Proof. Since $w-v \in W_{0}^{1, \tilde{\varphi}}\left(B_{r}\right)$, we infer from (4.18) and (4.19) that

$$
\begin{aligned}
& \int_{B_{r}}\left[\frac{\tilde{\varphi}^{\prime}(|\nabla w|)}{|\nabla w|} \nabla w-\frac{\tilde{\varphi}^{\prime}(|\nabla v|)}{|\nabla v|} \nabla v\right] \cdot(\nabla w-\nabla v) d x \\
& =\int_{B_{r}}\left[\frac{\tilde{\varphi}^{\prime}(|\nabla \psi|)}{|\nabla \psi|} \nabla \psi-\frac{\tilde{\varphi}^{\prime}\left(\left|\nabla \psi\left(x_{0}\right)\right|\right)}{\left|\nabla \psi\left(x_{0}\right)\right|} \nabla \psi\left(x_{0}\right)\right] \cdot(\nabla w-\nabla v) d x,
\end{aligned}
$$

where $x_{0}$ is the center of $B_{r}$. In a similar way as in the estimation of $I_{3}$ in the previous lemma, we see that

$$
\begin{aligned}
& f_{B_{r}}\left[\frac{\tilde{\varphi}^{\prime}(|\nabla \psi|)}{|\nabla \psi|} \nabla \psi-\frac{\tilde{\varphi}^{\prime}\left(\left|\nabla \psi\left(x_{0}\right)\right|\right)}{\left|\nabla \psi\left(x_{0}\right)\right|} \nabla \psi\left(x_{0}\right)\right] \cdot(\nabla w-\nabla v) d x \\
& \lesssim r^{\beta}\left(f_{B_{r}} \tilde{\varphi}(|\nabla u|) d x+1\right) .
\end{aligned}
$$


Therefore, by Lemma 2.10 (2) \& (4) with (4.21) and (4.22), we obtain that

$$
\begin{aligned}
& f_{B_{r}} \tilde{\varphi}(|\nabla w-\nabla v|) d x \\
& \lesssim \kappa\left(f_{B_{r}} \tilde{\varphi}(|\nabla u|) d x+1\right)+\kappa^{-1} r^{\beta}\left(f_{B_{r}} \tilde{\varphi}(|\nabla u|) d x+1\right),
\end{aligned}
$$

which implies that

$$
f_{B_{r}} \tilde{\varphi}(|\nabla w-\nabla v|) d x \lesssim r^{\frac{\beta}{2}}\left[\tilde{\varphi}\left(f_{B_{2 r}}|\nabla u| d x\right)+1\right]
$$

by taking $\kappa:=r^{\frac{\beta}{2}}$. With Jensen's inequality, $(\mathrm{aDec})_{q}$ and increasingness of $\tilde{\varphi}$, the claim follows in the same way as in the proof of the previous lemma.

The following is the well-known technical iteration lemma (cf. [36, Lemma 7.1] and [26, Lemma 2.1, Chapter 3]).

Lemma 4.39. Let $g:\left[0, r_{0}\right] \rightarrow[0, \infty)$ be a non-decreasing function. Suppose that

$$
g(\varrho) \leqslant C\left[\left(\frac{\varrho}{r}\right)^{n}+\epsilon\right] g(r)+C r^{n}
$$

for all $0<\varrho<r \leqslant r_{0}$ with non-negative constant $C$. Then for any $\mu \in(0, n)$ there exist $\epsilon_{1}=\epsilon_{1}(n, C, \mu)>0$ such that if $\epsilon<\epsilon_{1}$, we have

$$
g(\varrho) \leqslant c\left(\frac{\varrho}{r}\right)^{n-\mu}\left(g(r)+r^{n-\mu}\right)
$$

where $c$ is a constant depending on $n, C$ and $\mu$.

Now we prove our main results. In the results, $\omega$ is the function from (wVA1) for $\epsilon=\epsilon_{0}$ and $L \geqslant 1$ is the constant from (A0). We also remark that (wVA1) can be replaced by the conditions (A1) and (wVA1) with fixed $\epsilon>0$ which is sufficiently small depending on $n, p, q, L$.

Proof of Theorem $2.7(i)$. Let $r_{0} \in(0,1)$ be a sufficiently small number which will be determined later. Consider any $B_{r_{0}} \subset \Omega^{\prime} \Subset \Omega$ assuming that $r_{0}>0$ satisfies (4.10) with $r=r_{0}$, and let $0<2 r \leqslant r_{0}$.

First we recall Jensen's inequality and (4.22) followed by Lemma 4.16 (3) and (4.30) to obtain

$$
\begin{aligned}
\tilde{\varphi}\left(f_{B_{r}}|\nabla v| d x\right) & \leqslant f_{B_{r}} \tilde{\varphi}(|\nabla v|) d x \lesssim f_{B_{r}} \tilde{\varphi}(|\nabla u|) d x+1 \\
& \lesssim\left(f_{B_{r}} \varphi(x,|\nabla u|)^{1+\sigma_{0}} d x\right)^{\frac{1}{1+\sigma_{0}}}+1 \lesssim \tilde{\varphi}\left(f_{B_{2 r}}|\nabla u| d x\right)+1
\end{aligned}
$$

Now if we apply $\tilde{\varphi}^{-1}$ to both sides of the inequality and use (aDec) to insert the " +1 " inside the $\Phi$-function we get

$$
f_{B_{r}}|\nabla v| d x \lesssim f_{B_{2 r}}|\nabla u|+1 d x
$$


For simplicity, we write $\omega_{0}(r):=\left(\omega(2 r)^{p / q}+r^{\gamma}+r^{\beta}\right)^{\frac{1}{2 q}}$. Then if $\varrho<\frac{r}{2}$, by Lemmas 4.32,4.38 and (4.23), we have that

$$
\begin{aligned}
\int_{B_{\varrho}}|\nabla u| d x & \leqslant \int_{B_{r}}|\nabla u-\nabla w| d x+\int_{B_{r}}|\nabla w-\nabla v| d x+\int_{B_{\varrho}}|\nabla v| d x \\
& \lesssim \omega_{0}(r) \int_{B_{2 r}}|\nabla u|+1 d x+\varrho^{n} \sup _{B_{r / 2}}|\nabla v| \\
& \lesssim \omega_{0}\left(r_{0}\right) \int_{B_{2 r}}|\nabla u|+1 d x+\varrho^{n} f_{B_{r}}|\nabla v| d x \\
& \lesssim\left(\omega_{0}\left(r_{0}\right)+\left(\frac{\varrho}{r}\right)^{n}\right) \int_{B_{2 r}}|\nabla u| d x+r^{n} .
\end{aligned}
$$

Otherwise, i.e. if $\frac{r}{2} \leqslant \varrho<2 r$, the above estimate is clear because $\frac{1}{2} \leqslant \frac{\varrho}{r}$.

Therefore, by choosing $r_{0}$ so small that $\omega_{0}\left(r_{0}\right)=\left(\omega\left(r_{0}\right)+r_{0}^{\gamma}+r_{0}^{\beta}\right)^{\frac{1}{2 q}}<\epsilon_{1}$, where $\epsilon_{1}$ is given in Lemma 4.39, we have from Lemma 4.39 that for any $\mu \in(0, n)$,

$$
\int_{B_{\varrho}}|\nabla u| d x \lesssim\left(\frac{\varrho}{r_{0}}\right)^{n-\mu}\left(\int_{B_{r_{0}}}|\nabla u| d x+r_{0}^{n-\mu}\right)
$$

for all $B_{\varrho} \subset \Omega^{\prime}$ with $\varrho \in\left(0, r_{0}\right)$. In addition, $B_{\varrho} \subset \Omega^{\prime}$ is arbitrary and the implicit constant is universal. Hence, we take $1-\mu=\alpha$ to obtain that $u \in C_{\text {loc }}^{0, \alpha}\left(\Omega^{\prime}\right)$ by Morrey type embedding.

Proof of Theorem 2.7 (ii). Fix $\Omega^{\prime} \Subset \Omega$. Recall from (4.24) that for any $0<\varrho<\frac{r}{2}$

$$
f_{B_{\varrho}}\left|\nabla v-(\nabla v)_{B_{\varrho}}\right| d x \leqslant c\left(\frac{\varrho}{r}\right)^{\alpha_{0}} f_{B_{r / 2}}|\nabla v| d x
$$

for some $\alpha_{0}>0$. From (4.41), we note that for $\mu \in(0,1)$,

$$
f_{B_{2 r}}|\nabla u| d x \leqslant c_{\mu} r^{-\mu}
$$

for all $B_{2 r} \subset \Omega^{\prime}$ with $r \in\left(0, \frac{r_{0}}{2}\right]$, where $r_{0}$ is from the proof of Theorem 2.7 (i) and the constant $c_{\mu} \geqslant 1$ depends on $n, p, q, L, r_{0}$, and $\mu$. Let us consider a small $r<\frac{r_{0}}{2}$ which will be determined later. From Lemmas 4.32, 4.38 and (4.40), we then derive that for $0<\varrho<\frac{r}{2}$,

$$
\begin{aligned}
& f_{B_{\varrho}}\left|\nabla u-(\nabla u)_{B_{\varrho}}\right| d x \leqslant 2 f_{B_{\varrho}}\left|\nabla u-(\nabla v)_{B_{\varrho}}\right| d x \\
& \quad \leqslant 2 f_{B_{\varrho}}|\nabla u-\nabla w| d x+2 f_{B_{\varrho}}|\nabla w-\nabla v| d x+2 f_{B_{\varrho}}\left|\nabla v-(\nabla v)_{B_{\varrho}}\right| d x \\
& \quad \lesssim\left(\frac{r}{\varrho}\right)^{n} f_{B_{r}}|\nabla u-\nabla w| d x+\left(\frac{r}{\varrho}\right)^{n} f_{B_{r}}|\nabla w-\nabla v| d x+\left(\frac{\varrho}{r}\right)^{\alpha_{0}} f_{B_{r / 2}}|\nabla v| d x \\
& \quad \lesssim\left(\frac{r}{\varrho}\right)^{n}\left(\omega(2 r)^{p / q}+r^{\gamma}+r^{\beta}\right)^{\frac{1}{2 q}}\left(f_{B_{2 r}}|\nabla u| d x+1\right)+\left(\frac{\varrho}{r}\right)^{\alpha_{0}} f_{B_{r / 2}}|\nabla v| d x \\
& \quad \lesssim\left(r^{\delta_{0}}\left(\frac{r}{\varrho}\right)^{n}+\left(\frac{\varrho}{r}\right)^{\alpha_{0}}\right)\left(f_{B_{2 r}}|\nabla u| d x+1\right) \leqslant c_{\mu} r^{-\mu}\left(r^{\delta_{0}}\left(\frac{r}{\varrho}\right)^{n}+\left(\frac{\varrho}{r}\right)^{\alpha_{0}}\right)
\end{aligned}
$$

where $\delta_{0}:=\frac{1}{2 q} \min \left\{\frac{\delta p}{q}, \gamma, \beta\right\}$. In the same way as in the proof of Theorem 7.4 in [36], we obtain $\nabla u \in C_{\text {loc }}^{\alpha}\left(\Omega^{\prime}\right)$ for some $\alpha>0$. 


\section{REFERENCES}

[1] E. Acerbi and G. Mingione: Regularity results for a class of functionals with nonstandard growth, Arch. Ration. Mech. Anal. 156 (2) (2001) 121-140.

[2] W. Arriagada and J. Huentutripay: Improved bounds for solutions of $\varphi$-Laplacians, Opuscula Math. 38, no. 6 (2018), 765-777.

[3] P. Baroni, M. Colombo and G. Mingione: Non-autonomous functionals, borderline cases and related function classes, St Petersburg Math. J. 27 (2016), 347-379.

[4] P. Baroni, M. Colombo and G. Mingione: Regularity for general functionals with double phase, Calc. Var. Partial Differential Equations, Volume 57, no. 2, 62 (2018).

[5] H. Brézis and D. Kinderlehrer: The smoothness of solutions to nonlinear variational inequalities, Indiana Univ. Math. J. 23 (1973-1974), 831-844 .

[6] M. Caselli, M. Eleuteri and A. P. di Napoli: Regularity results for a class of obstacle problems with $p, q$-growth conditions, ESAIM Control Optim. Calc. Var., to appear. DOI: $10.1051 /$ cocv/2021017

[7] L.A. Caffarelli and D. Kinderlehrer: Potential methods in variational inequalities. J. Analyse Math. 37 (1980), 285-295.

[8] I. Chlebicka and C. De Filippis: Removable sets in non-uniformly elliptic problems. Annali di Matematica 199, 619-649 (2020).

[9] H. Choe: A regularity theory for a general class of quasilinear elliptic partial differential equations and obstacle problems. Arch. Rational Mech. Anal. 114 (1991), no. 4, 383-394.

[10] H. Choe and J.L. Lewis: On the obstacle problem for quasilinear elliptic equations of $p$-Laplacian type. SIAM J. Math. Anal. 22 (1991), no. 3, 623-638.

[11] A. Cianchi: Boundedness of solutions to variational problems under general growth conditions. Comm. Partial Differential Equations, 22(9-10):1629-1646, 1997.

[12] A. Cianchi and N. Fusco: Gradient regularity for minimizers under general growth conditions. J. Reine Angew. Math., 507:15-36, 1999.

[13] M. Colombo and G. Mingione: Regularity for double phase variational problems, Arch. Ration. Mech. Anal. 215 (2015), no. 2, 443-496.

[14] A. Coscia and G. Mingione: Hölder continuity of the gradient of $p(x)$-harmonic mappings, C. R. Acad. Sci. Paris, Sér. I 328 (1999), 363-368.

[15] G. Cupini, F. Giannetti, R. Giova and A. Passarelli di Napoli: Regularity results for vectorial minimizers of a class of degenerate convex integrals, J. Differential Equations 265 (2018), no. 9, 4375-4416

[16] C. De Filippis: Regularity results for a class of non-autonomous obstacle problems with $(p, q)$-growth, J. Math. Anal. Appl. (2019), Doi: 10.1016/j.jmaa.2019.123450

[17] C. De Filippis and J. Oh: Regularity for multi-phase variational problems, J. Differential Equations 267 (2019), no. 3, 1631-1670.

[18] L. Diening, B. Stroffolini and A. Verde: Everywhere regularity of functionals with $\varphi$-growth, manuscripta math. 129, 449-481 (2009).

[19] M. Eleuteri: Hölder continuity results for a class of functionals with non-standard growth. Boll. Unione Mat. Ital. Sez. B Artic. Ric. Mat. (8), 7 (1), (2004), 129-157.

[20] M. Eleuteri and J. Habermann: Regularity results for a class of obstacle problems under nonstandard growth conditions. J. Math. Anal. Appl. 344 (2008), no. 2, 1120-1142.

[21] M. Eleuteri and J. Habermann: A Hölder continuity result for a class of obstacle problems under non standard growth conditions. Math. Nachr. 284 (2011), no. 11-12, 1404-1434.

[22] M. Eleuteri, P. Harjulehto and T. Lukkari: Global regularity and stability of solutions to obstacle problems with nonstandard growth, Rev. Mat. Complut. 26 (2013), 147-181.

[23] X. Fan, Global $C^{1, \alpha}$ regularity for variable exponent elliptic equations in divergence form, J. Differ. Equ. 235 (2) (2007), 397-417.

[24] M. Fuchs: Hölder continuity of the gradient for degenerate variational inequalities. Nonlinear Anal. 15 (1990), no. 1, 85-100.

[25] M. Fuchs and G. Mingione: Full $C^{1, \alpha}$-regularity for free and constrained local minimizers of elliptic variational integrals with nearly linear growth. Manuscr. Math. 102 (2000), 227-250.

[26] M. Giaquinta: Multiple Integrals in the Calculus of Variations and Nonlinear Elliptic Systems, Annals of Mathematics Studies, vol.105, Princeton University Press, Princeton, NJ, 1983.

[27] M. Giaquinta and E. Giusti: Differentiability of minima of non-differentiable functionals, Invent. Math. 72 (1983), 285-298. 
[28] F. Giannetti and A. Passarelli di Napoli: Regularity results for a new class of functionals with non-standard growth conditions, J. Differential Equations 254 (2013) 1280-1305.

[29] E. Giusti: Direct Methods in the Calculus of Variations, World Scientific, Singapore, 2003.

[30] P. Harjulehto and P. Hästö: Orlicz spaces and Generalized Orlicz spaces, Lecture Notes in Mathematics, vol. 2236, Springer, Cham, 2019, X+168.

[31] P. Harjulehto, P. Hästö and R. Klén: Generalized Orlicz spaces and related PDE, Nonlinear Anal. 143 (2016), 155-173.

[32] P. Harjulehto, P. Hästö and A. Karppinen: Local higher integrability of the gradient of a quasiminimizer under generalized Orlicz growth conditions, Nonlinear Anal. Volume 177, Part B, December 2018, Pages 543-552.

[33] P. Harjulehto, P. Hästö and M. Lee: Hölder continuity of quasiminimizers and $\omega$-minimizers of functionals with generalized Orlicz growth, Ann. Sc. Norm. Super. Pisa Cl. Sci., to appear. DOI:10.2422/20362145.201908_015

[34] P. Harjulehto, P. Hästö, V. Latvala and O. Toivanen: Critical variable exponent functionals in image restoration, Appl. Math. Letters 26 (2013), 56-60.

[35] P. Harjulehto, P. Hästö and O. Toivanen: Hölder regularity of quasiminimizers under generalized growth conditions, Calc. Var. Partial Differential Equations 56 (2017), no. 2, Art. 22, 26 pp.

[36] P. Hästö and J. Ok: Maximal regularity for non-autonomous functionals, J. Eur. Math. Soc., to appear.

[37] G.M. Lieberman: The natural generalization of the natural conditions of Ladyzhenskaya and Ural'tseva for elliptic equations, Comm. Partial Differential Equations 16 (1991), no. 2-3, 311-361.

[38] T. Kilpeläinen, X. Zhong: Removable set for continuous solutions of quasilinear elliptic equations, Proc. Amer. Math. Soc. 130 (2002), no. 6, 1681-1688.

[39] G. M. Lieberman: On the natural generalization of the natural conditions of Ladyzhenskaya and Ural'tseva, Comm. Partial Differential Equations 16 (1991), no. 2-3, 311-361.

[40] P. Marcellini: Regularity of minimizers of integrals of the calculus of variations with nonstandard growth conditions, Arch. Rational Mech. Anal. 105 (1989), no. 3, 267-284.

[41] P. Marcellini: Regularity and existance of solutions of elliptic equations with $p, q$-growth conditions, J. Differential Equations 50 (1991), no. 1, 1-30.

[42] T. Mengesha and N.C. Phuc: Global estimates for quasilinear elliptic equations on Reifenberg flat domains, Arch. Ration. Mech. Anal. 203 (2012) 189-216.

[43] J.H. Michael and W.P. Ziemer: Interior regularity for solutions to obstacle problems. Nonlinear Anal. 10 (1986), no. 12, 1427-1448.

[44] G. Mingione: Regularity of minima: An invitation to the dark side of the calculus of variations, Appl. Math. 51 (2006), no. 4, 355-426.

[45] G. Mingione and F. Siepe: Full $C^{1, \alpha}$-regularity for minimizers of integral functionals with $L \log L$ growth. Z. Anal. Anwend. 18 (1999) 1083-1100.

[46] J. Mu and W.P. Ziemer: Smooth regularity of solutions of double obstacle problems involving degenerate elliptic equations. Commun. Partial Differ. Equ. 16(4-5) (1991), 821-843.

[47] J. Ok: Regularity results for a class of obstacle problems with nonstandard growth, J. Math. Anal. Appl. 444 (2016), no. 2, 957-979.

[48] J. Ok: Gradient continuity for nonlinear obstacle problems. Mediterr. J. Math. 14 (2017), no. 1, Paper No. $16,24 \mathrm{pp}$.

[49] J. Ok: Harnack inequality for a class of functionals with non-standard growth via De Giorgi's method. Adv. Nonlinear Anal. 7(2), 167-182 (2018)

[50] M.A. Ragusa and A. Tachikawa: Regularity for minimizers for functionals of double phase with variable exponents, Adv. Nonlinear Anal. 9 (2020), no. 1, 710-728.

[51] V.V. Zhikov: On Lavrentiev's Phenomenon, Russian J. Math. Phys. 3 (1995), 249-269.

[52] V.V. Zhikov: On some variational problems. Russian J. Math. Phys., 5 (1997), 105-116

\section{A. KARPPINEN}

Department of Mathematics and Statistics, FI-20014 University of Turku, Finland

arttu.a.karppinen@utu.fi

M. LEE

Department of Mathematics, Pusan National University, Busan 46241, Republic of Korea

mikyounglee@pusan.ac.kr 